MATHEMATICS OF COMPUTATION

Volume 76, Number 257, January 2007, Pages 19-42

S $0025-5718(06) 01879-5$

Article electronically published on October 4, 2006

\title{
LOCAL A POSTERIORI ESTIMATES FOR POINTWISE GRADIENT ERRORS \\ IN FINITE ELEMENT METHODS FOR ELLIPTIC PROBLEMS
}

\author{
ALAN DEMLOW
}

\begin{abstract}
We prove local a posteriori error estimates for pointwise gradient errors in finite element methods for a second-order linear elliptic model problem. First we split the local gradient error into a computable local residual term and a weaker global norm of the finite element error (the "pollution term"). Using a mesh-dependent weight, the residual term is bounded in a sharply localized fashion. In specific situations the pollution term may also be bounded by computable residual estimators. On nonconvex polygonal and polyhedral domains in two and three space dimensions, we may choose estimators for the pollution term which do not employ specific knowledge of corner singularities and which are valid on domains with cracks. The finite element mesh is only required to be simplicial and shape-regular, so that highly graded and unstructured meshes are allowed.
\end{abstract}

\section{INTRODUCTION AND RESULTS}

We consider finite element approximations to the second-order linear elliptic model problem

$$
\begin{aligned}
-\operatorname{div}(A \nabla u) & =f \text { in } \Omega \\
u & =0 \text { on } \partial \Omega
\end{aligned}
$$

where $\Omega$ is a bounded domain in $\mathbb{R}^{n}$ with $n \geq 2, A \in\left[W_{\infty}^{1}(\Omega)\right]^{n \times n}$, and $f \in L_{\infty}(\Omega)$. We shall particularly concentrate on the case where $n=2$ or $n=3$ and $\Omega$ is polygonal or polyhedral, and will assume that this is the case unless otherwise noted. Let $\mathcal{T}$ be a shape-regular simplicial decomposition of $\Omega$. No further restrictions are placed on $\mathcal{T}$, so that highly graded and unstructured meshes are admitted. We then take $S_{h}^{r}$ to be the space of continuous functions which are piecewise polynomials of degree $r-1$ on $\mathcal{T}$ and which are 0 on $\partial \Omega$. Finally, let $u_{h} \in S_{h}^{r}$ be the standard Galerkin approximation to $u$, that is,

$$
\int_{\Omega} A \nabla u_{h} \cdot \nabla \chi \mathrm{d} x=\int_{\Omega} f \chi \mathrm{d} x \forall \chi \in S_{h}^{r} .
$$

Received by the editor December 10, 2004 and, in revised form, September 16, 2005.

2000 Mathematics Subject Classification. Primary 65N30, 65N15.

Key words and phrases. Finite element methods, elliptic problems, a posteriori error estimation, local error analysis, pointwise error analysis.

This material is based upon work partially supported under a National Science Foundation postdoctoral research fellowship.

(C)2006 American Mathematical Society Reverts to public domain 28 years from publication 
In this paper we provide sharp residual-based a posteriori upper bounds for $\left\|\nabla\left(u-u_{h}\right)\right\|_{L_{\infty}(D)}$, where $D \subset \Omega$. These results are local counterparts to the localized (or weighted) a posteriori estimates for $\left\|\nabla\left(u-u_{h}\right)\right\|_{L_{\infty}(D)}$, which were proved in [De] when $\Omega$ is a convex polyhedron. Here we consider three cases: $D \subset \subset \Omega, D$ abuts a flat portion of $\partial \Omega$, and $D$ abuts $\partial \Omega$ but is sufficiently removed from nonconvex singular points (e.g., re-entrant corners) of $\partial \Omega$. Note that the second case includes subdomains $D$ which lie on one side of a crack in $\Omega$. We follow the nomenclature of [Da88] and say that $\Omega$ has a crack or slit if interior $(\bar{\Omega}) \backslash \Omega$ has codimension 1 or 2 , respectively.

Efficiently controlling local finite element errors generally involves bounding two quantities, a local approximation error and a global pollution term which measures the finite element error in a weaker norm. A priori estimates using this fundamental idea to bound the local energy error first appeared in [NS74. The current work is a maximum-norm counterpart to the recent paper [LN03], where estimators for the local energy norm which control both the local approximation error and the global pollution term a posteriori are analyzed. Our estimates are also a posteriori analogs to the weighted local a priori $W_{\infty}^{1}$ estimates proved in [Sch00]. Assuming that the mesh is quasi-uniform with diameter $h$ on $B_{d}\left(x_{0}\right)$, let

$$
\sigma_{x_{0}}(y)=\frac{h}{\left|x_{0}-y\right|+h} .
$$

Also, for $1 \leq p \leq \infty, \frac{1}{p}+\frac{1}{q}=1$, and $D \subset \Omega$, let

$$
\|v\|_{W_{p}^{-k}(D)}=\sup _{\|\psi\|_{W_{q}^{k}(D)}=1, \psi=0 \text { on } \partial D \backslash \partial \Omega}(v, \psi) .
$$

It was shown in $\left[\mathrm{Sch} 00\right.$ that if $A$ is sufficiently smooth on $B_{d}\left(x_{0}\right), \operatorname{dist}\left(x_{0}, \partial \Omega\right)>d$, and $d \geq C h$ for $C$ sufficiently large, then

$$
\begin{aligned}
& \left|\nabla\left(u-u_{h}\right)\left(x_{0}\right)\right| \\
& \leq C\left[\left(\ln \frac{d}{h}\right)^{\bar{s}} \min _{\chi \in S_{h}^{r}}\left(\left\|\sigma_{x_{0}}^{s} \nabla(u-\chi)\right\|_{L_{\infty}\left(B_{d}\left(x_{0}\right)\right)}+\frac{1}{d}\left\|\sigma_{x_{0}}^{s}(u-\chi)\right\|_{L_{\infty}\left(B_{d}\left(x_{0}\right)\right)}\right)\right. \\
& \left.\quad+d^{-1-k-\frac{n}{p}}\left\|u-u_{h}\right\|_{W_{p}^{-k}\left(B_{d}\left(x_{0}\right)\right)}\right] .
\end{aligned}
$$

Here $0 \leq s \leq r-1,1 \leq p \leq \infty, k \geq 0$, and $\bar{s}=1$ if $s=r-1$ and $\bar{s}=0$ otherwise. Note that the maximum exponent $s$ of the weight $\sigma_{x_{0}}$ increases as $r$ increases, so that the local approximation term

$$
\min _{\chi \in S_{h}^{r}}\left(\left\|\sigma_{x_{0}}^{s} \nabla(u-\chi)\right\|_{L_{\infty}\left(B_{d}\left(x_{0}\right)\right)}+\frac{1}{d}\left\|\sigma_{x_{0}}^{s}\left(u-u_{h}\right)\right\|_{L_{\infty}\left(B_{d}\left(x_{0}\right)\right)}\right)
$$

is more localized to the point $x_{0}$ in higher-order methods.

Before stating our a posteriori results, we introduce further notation. Let $h_{T}=$ $|T|^{1 / n}$ for $T \in \mathcal{T}$, and let $\underline{h}=\min _{T \in \mathcal{T}} h_{T}$ and $\bar{h}=\max _{T \in \mathcal{T}} h_{T}$. For a subset $D$ of $\Omega$, we define $\mathcal{T}_{D}=\{T \in \mathcal{T}: \bar{T} \cap D \neq \emptyset\}$ and $D^{h}=\bigcup_{T \in \mathcal{T}_{D}} T$. For $d>0$, we let $D_{d}$ be the union of all components of $\{x \in \Omega$ : $\operatorname{dist}(x, D)<d\}$ which have nontrivial intersection with $D$. Analogous to (1.3), we also define the piecewise-constant weight function

$$
\sigma_{D}(T)=\frac{h_{T}}{\operatorname{dist}(D, T)+h_{T}}
$$


Letting $S$ be a face shared by two elements $T_{1}$ and $T_{2}$ and letting $\vec{n}$ be a unit normal on $S$ (with arbitrary orientation), we next define for $v_{h} \in S_{h}^{r}$ and $x \in S$

$$
\left[A \nabla v_{h}\right](x)=A\left(\left.\nabla v_{h}\right|_{T_{1}}-\left.\nabla v_{h}\right|_{T_{2}}\right) \cdot \vec{n} .
$$

Finally we define the first-order elementwise maximum norm residual

$$
\mathcal{E}_{T}=h_{T}\left\|f+\operatorname{div}\left(A \nabla u_{h}\right)\right\|_{L_{\infty}(T)}+\left\|\left[A \nabla u_{h}\right]\right\|_{L_{\infty}(\partial T)} .
$$

We first prove the following a posteriori counterpart to the a priori interior result (1.4). Our estimate is also valid up to flat portions of $\partial \Omega$.

Theorem 1.1. Let $\Omega$ be an arbitrary domain in $\mathbb{R}^{n}$, where $n \geq 2$. Assume that $D \subset \Omega$ and $d>0$ are such that either $\operatorname{dist}(D, \partial \Omega)>d$ or $\operatorname{dist}(D, \partial \Omega) \leq d$, and $\{x \in \partial \Omega: \operatorname{dist}(x, D)<d\}$ lies in a single $n-1$-dimensional hyperplane. Assume that $A$ is sufficiently smooth on $D_{d}$ and that $u \in C^{1, \alpha}\left(\overline{D_{\rho}}\right)$ for some $0<\alpha \leq 1$, where $\rho \leq \min \left(\underline{h}, \frac{d}{8}\right)$. Then for any $k \geq 0$ and $1 \leq p \leq \infty$,

$$
\begin{aligned}
\left\|\nabla\left(u-u_{h}\right)\right\|_{L_{\infty}(D)} \leq & C_{\mathrm{app}}(r) \ell_{\rho, d} \max _{T \in \mathcal{T}_{D_{d}}} \sigma_{D}(T)^{r-1} \mathcal{E}_{T} \\
& +C_{\mathrm{pol}} d^{-1-k-\frac{n}{p}}\left\|u-u_{h}\right\|_{W_{p}^{-k}\left(D_{d}\right)}+C \rho^{\alpha}|u|_{C^{1, \alpha}\left(\overline{D_{\rho}}\right)} .
\end{aligned}
$$

Here $C_{\mathrm{pol}}$ depends on $A$ and $k, C_{\mathrm{app}}(r)$ depends on $A$, the shape regularity of $\mathcal{T}$, and $r$, and $\ell_{\rho, d}=\ln \frac{d}{\rho}$.

We note that Theorem 1.1 is valid independent of the boundary conditions under consideration when $D_{d} \subset \subset \Omega$.

The third term $C \rho^{\alpha}|u|_{C^{1, \alpha}\left(\overline{\left.D_{\rho}\right)}\right.}$ in (1.5) arises from regularizing the $\delta$-distribution in our proofs. Choosing $\rho=\underline{h}^{\beta}$ for $\beta \geq 1$ yields $C \rho^{\alpha}|u|_{C^{1, \alpha}\left(\overline{D_{\rho}}\right)}=C \underline{h}^{\alpha \beta}|u|_{C^{1, \alpha}\left(\overline{D_{\underline{h}}}\right)}$, so we may always ensure that this term is of higher order in the minimum mesh diameter by choosing $\beta$ large enough. In addition, it may be reabsorbed into the left-hand side under the nondegeneracy assumption

$$
\left\|\nabla\left(u-u_{h}\right)\right\|_{L_{\infty}\left(D_{\rho}\right)} \geq C \underline{h}^{\epsilon}|u|_{C^{1, \alpha}\left(\overline{D_{\rho}}\right)}
$$

for some $\epsilon>0$. In the following corollary, we show that if $u$ satisfies a certain smoothness and nondegeneracy assumption in a neighborhood of a single point in $D$, then (1.6) holds (in slightly modified form) and we may in fact eliminate this regularization penalty.

Corollary 1.2. Assume that $D, d$, and $\Omega$ satisfy the geometric conditions of Theorem 1.1 and that $A$ is sufficiently smooth on $D_{d}$. In addition, assume that there exist a point $x_{1} \in D$ and an $\eta>0$ such that $\left|D^{\gamma} u\left(x_{1}\right)\right| \geq C^{*}>0$ for some multi-index $\gamma$ with $|\gamma|=r$ and $\|u\|_{W_{\infty}^{r+1}\left(B_{\eta}\left(x_{1}\right)\right)} \leq C^{* *}$. Finally, assume that $u \in C^{1, \alpha}\left(\overline{D_{\tilde{\eta}}}\right)$ for some $0<\alpha<1$ and $\tilde{\eta}>0$. Then

$$
\begin{aligned}
\left\|\nabla\left(u-u_{h}\right)\right\|_{L_{\infty}(D)} \leq & C_{\text {app }}(r) \tilde{\ell}_{\underline{h}, d} \max _{T \in \mathcal{T}_{D_{d}}} \sigma_{D}(T)^{r-1} \mathcal{E}_{T} \\
& +C_{\mathrm{pol}} d^{-1-k-\frac{n}{p}}\left\|u-u_{h}\right\|_{W_{p}^{-k}\left(D_{d}\right)} .
\end{aligned}
$$

Here

$$
\tilde{\ell}_{\underline{h}, d}=\left|\ln \left[\min \left(\frac{1}{d}\left(\frac{C^{*}}{C^{* *}+C(D, d)\|f\|_{L_{\infty}(\Omega)}}\right)^{\frac{r}{\alpha}}, \frac{h^{\frac{r}{\alpha}}}{d}, \frac{\eta^{\frac{r}{\alpha}}}{d}, \frac{\tilde{\eta}^{\frac{r}{\alpha}}}{d}\right)\right]\right| .
$$


For polynomial degree $r-1=1$ the smoothness assumption of Corollary 1.2 essentially appears in [BM87] (in the $H^{1}$ context) and [Noc95] (in the $L_{\infty}$ context). There the mesh is required to resolve the ball $B_{\eta}\left(x_{1}\right)$, leading to a posteriori statements which only have asymptotic validity. Here we decouple this nondegeneracy condition from the finite element mesh and thus gain an estimate which is valid on coarse meshes. The price we pay for this improvement is the presence of the logarithmic factor $\tilde{\ell}_{\underline{h}, d}$ which depends on a priori quantities in the pre-asymptotic range.

The smoothness and nondegeneracy assumption of Corollary 1.2 is met in many, but not all, practical situations. If $D$ contains a subdomain of $\Omega$, then $u$ will generally be smooth on some portion of that subdomain unless $A$ or $f$ is pathological. A simple example where $u$ is not sufficiently smooth on $D$ occurs when $f$ is piecewise constant and $D$ is a line segment across which $f$ has a jump. Here $u$ has Hölder continuous first derivatives on $D$ but generally not a higher degree of smoothness. This situation may be remedied by taking $D$ to be a small neighborhood of the line segment in question so that $f$ is smooth on some subdomain of $D$. Note also that the a priori-dependent higher order term $C \rho^{\alpha}|u|_{C^{1, \alpha}\left(\overline{D_{\rho}}\right)}$ in (1.5) is removed in (1.7), but at the expense of the constant $\tilde{\ell}_{\underline{h}, d}$ on the right-hand side which depends on $u$ and $f$ in the pre-asymptotic range. However, $\tilde{\ell}_{\underline{h}, d}$ is only logarithmically (and thus weakly) dependent upon $u$ and $f$. In addition,

$$
\tilde{\ell}_{\underline{h}, d}=\ln \frac{d}{\underline{h}^{\frac{r}{\alpha}}}
$$

when

$$
\underline{h} \leq \min \left(\tilde{\eta}, \eta, \frac{C^{*}}{C^{* *}+C(D, d)\|f\|_{L_{\infty}(\Omega)}}\right) .
$$

Ignoring the factor $\tilde{\ell}_{\underline{h}, d}$ is thus very similar to ignoring factors of $\ln \frac{1}{\underline{h}}$, which is routinely done in computational practice.

We emphasize that (1.5) and (1.7) hold on very coarse meshes. Note especially that in contrast to both the a priori estimate (1.4) and the local a posteriori results of [LN03, Theorem 1.1 and Corollary 1.2 do not require a priori that the mesh resolve the layer $D_{d} \backslash D$. Refinement algorithms using (1.5) will however naturally tend to resolve $D_{d} \backslash D$.

We next discuss the two terms on the right-hand side of (1.7). The first term $\max _{T \in \mathcal{T}_{D_{d}}} \sigma_{D}(T)^{r-1} \mathcal{E}_{T}$ in (1.7) corresponds to the local approximation error over $D_{d}$. Note that $\sigma_{D} \equiv 1$ on $D^{h}$, and $\sigma_{D}(T) \approx h(T)$ if $\operatorname{dist}(T, D)$ is of unit size. Thus using the weight $\sigma_{D}$ we are able to bound this local residual term in an increasingly sharp fashion as the polynomial order $r-1$ is increased.

The second term $d^{-1-k-\frac{n}{p}}\left\|u-u_{h}\right\|_{W_{p}^{-k}\left(D_{d}\right)}$ in (1.7) measures the pollution effect of the solution from outside of $D$. In order to gain a computable estimator from (1.7), it is necessary to bound this term a posteriori. In principle it is advantageous to measure the pollution error in the weakest norm possible. However, conveniently bounding negative norms of $u-u_{h}$ generally requires that $\partial \Omega$ and $A$ be relatively smooth, in which case one may in principle instead prove a global weighted (localized) estimate similar to that proved in [De. It should also be noted that rigorous adaptive strategies for problems on curved domains have only been investigated in limited cases which do not include either maximum norm estimators or negative norm estimators; cf. DR98 for the energy and $L_{2}$ norms. 
When $\Omega$ is a (possibly nonconvex) polyhedral domain in $\mathbb{R}^{2}$ or $\mathbb{R}^{3}$ and $A=I$, several possibilities exist for bounding the pollution term in (1.7). In $\mathbb{R}^{2}$, one may make use of precise knowledge of the singularities arising at re-entrant corners of $\partial \Omega$ to bound $\left\|u-u_{h}\right\|_{L_{2}(\Omega)}$ by a weighted residual estimator; cf. [LN03]. However, precise knowledge of singularities is often not available in $\mathbb{R}^{3}$. Here one may instead use residual-based bounds for $\left\|u-u_{h}\right\|_{L_{\infty}(\Omega)}$; cf. [Noc95], DDP00, and [NSSV]. Finally, for $p$ "large" but not $\infty$ (e.g., $p=5$ ), we may use the regularity results for general polyhedral domains given in Da92] to bound $\left\|u-u_{h}\right\|_{L_{p}(\Omega)}$ by a residual estimator which does not require specific knowledge of boundary singularities.

In computational experiments we considered the effectiveness of error estimators and indicators derived from (1.7) for controlling local gradient errors in plane polygonal crack domains via adaptive mesh refinement. The initial solution was computed on a coarse, uniform initial mesh with $\underline{h}=\bar{h} \approx 1$. The pollution error was measured in $L_{2}, L_{5}$, and $L_{\infty}$ using the residual estimators described in the preceding paragraph and using quadratic and cubic elements. When $d \approx 1$, measuring the pollution term in $L_{5}$ yielded the best error reduction with respect to the number of degrees of freedom, though all three options $\left(L_{2}, L_{5}\right.$, and $\left.L_{\infty}\right)$ were competitive. When $d \ll 1$ (for example, when $D$ is close to the crack tip), the factor $d^{-1-\frac{n}{p}}$ in (1.7) becomes relatively large, especially for $p=2$. In our tests the extra regularity gained from measuring the pollution term in the weaker $L_{2}$ norm instead of the $L_{\infty}$ or $L_{5}$ norm did not compensate for the extra negative power of $d$, and measuring the pollution term in $L_{\infty}$ or $L_{5}$ yielded a more efficient adaptive algorithm.

An outline of this note is as follows. In $\S 2$, we give further preliminaries and discussion of our assumptions. In $\S 3$ we prove Theorem 1.1 and Corollary 1.2. and state and prove results similar to (1.5) and (1.7) which also hold near convex corners of $\Omega$. In $\S 4$ we discuss in more detail a posteriori bounds for the pollution term in (1.5) and (1.7). In $\S 5$ we present some numerical examples on a plane crack domain.

\section{Preliminaries}

In this section we give some preliminary lemmas and definitions.

2.1. Finite element space and mesh. In addition to the definitions of the previous section, we first state a bound on the growth of the diameters of elements in shape-regular meshes; cf. De] for a proof. In the following proposition, we denote by $h(y)$ the quantity $h\left(T_{y}\right)$, where $y \in \bar{T}$.

Proposition 2.1. Assume that the triangulation $\mathcal{T}$ is shape-regular. Then there exists a constant $C_{\mathcal{T}}$ depending only on the shape-regularity of $\mathcal{T}$ such that for the barycenter $x_{T}$ of each element $T \in \mathcal{T}$, there holds for each point $y \in \Omega \backslash T$

$$
h(y) \leq C_{\mathcal{T}}\left|x_{T}-y\right| .
$$

Next we state approximation results. Let $P_{T}$ be the union of all elements sharing a vertex with $T$, let $P_{T}^{\prime}$ be the union of all elements sharing vertices with elements in $P_{T}$, and let $P_{T}^{\prime \prime}=\left(P_{T}^{\prime}\right)^{\prime}$. We shall employ the Scott-Zhang interpolation operator $I_{h}$ defined in SZ90] which preserves homogeneous boundary conditions on polyhedral 
domains and which for $1 \leq p \leq \infty$ satisfies

$$
\left\|v-I_{h} v\right\|_{L_{p}(T)} \leq C h_{T}^{j}\|v\|_{W_{p}^{j}\left(P_{T}\right)}, 1 \leq j \leq r
$$

and

$$
\left\|v-I_{h} v\right\|_{W_{p}^{1}(T)} \leq C h_{T}^{j}\|v\|_{W_{p}^{1+j}\left(P_{T}\right)}, 0 \leq j \leq r-1 .
$$

We shall actually apply $I_{h}$ in a slightly modified form. Assume that $v \in H_{0}^{1}(D)$ for some $D \subset \Omega$, and $\operatorname{dist}(\operatorname{supp}(v), \partial D \backslash \partial \Omega)>0$. We may define $I_{h}$ so that $\operatorname{supp}\left(I_{h} v\right) \subset D^{h}$. Indeed, doing so only requires that for each nodal point $a_{i} \in \partial D^{h}$, the face associated to $a_{i}$ in [SZ90] does not lie in the interior of $D^{h}$, a choice which may be made in the definition of $I_{h}$. $I_{h}$ then depends on $D$, but the constants $C$ in (2.2) and (2.3) do not.

In addition to the above interpolation results, we shall also employ the following lemma showing that $\left\|\nabla\left(u-u_{h}\right)\right\|_{L_{\infty}}$ may be bounded below as well as above. This result is proven in Lemma 3.3 of [HSWW01], though our statement of the lemma is slightly different. We let $\Pi^{r-1}=\left[P^{r-1}\right]^{n}$, where $P^{r-1}$ denotes the polynomials of degree $r-1$.

Lemma 2.2. Let $T_{1}$ be a shape-regular simplex of diameter $\tilde{\rho}$, and assume that $u \in W_{\infty}^{r+1}\left(T_{1}\right)$. Then

$$
\min _{\chi \in \Pi^{r-1}}\|\nabla(u-\chi)\|_{L_{\infty}\left(T_{1}\right)} \geq C\left(\tilde{\rho}^{r-1}|u|_{W_{\infty}^{r}\left(T_{1}\right)}-\tilde{\rho}^{r}\|u\|_{W_{\infty}^{r+1}\left(T_{1}\right)}\right) .
$$

Finally, we define local residuals which are useful for estimating $\left\|u-u_{h}\right\|_{W_{p}^{-k}(\Omega)}$. With $k \geq 0$ and $1 \leq p<\infty$, let

$$
\eta_{k, p}(T)^{p}=h_{T}^{p(2+k)}\left\|f+\operatorname{div}\left(A \nabla u_{h}\right)\right\|_{L_{p}(T)}^{p}+h_{T}^{p(k+1)+1}\left\|\left[A \nabla u_{h}\right]\right\|_{L_{p}(\partial T)}^{p} .
$$

2.2. Definition of reference domains. Our proofs involve carrying out duality arguments over subdomains of $\Omega$. When carrying out arguments near flat portions of $\partial \Omega$ and near the boundary of polyhedral domains, we shall need to carefully control the size and shape of these subdomains. We thus define reference domains to which we may scale portions of $\Omega$ lying near $\partial \Omega$.

In the case of polyhedral domains $\Omega$ in $\mathbb{R}^{2}$ or $\mathbb{R}^{3}$, one may construct a set $\left\{P_{1}, \ldots, P_{M-1}\right\}$ of polyhedron with the following properties:

(1) $\overline{P_{i}}$ contains at most one vertex of $\partial \Omega$. If $\overline{P_{i}}$ does not contain a vertex, then it intersects at most one edge. In addition, $\operatorname{diam}\left(P_{i}\right) \leq 1,1 \leq i \leq M_{1}$.

(2) There exist $c_{3}>0$ and $d_{0} \leq 1$ such that if $x \in \Omega$, dist $(x, \partial \Omega) \leq c_{3} d$, and $d \leq d_{0}$, then the following holds. There is a polyhedron $P_{i}$ such that $P_{i}$ is convex if and only if the component of $B_{2 d}(x) \cap \Omega$ containing $x$ is convex. In addition there is a copy $P_{x, d}$ of $P_{i}$ which is scaled by $d$, translated, and rotated so that $P_{x, d} \subset \Omega, x \in P_{x, d}$, and $\operatorname{dist}\left(x, \partial P_{x, d} \backslash \partial \Omega\right) \geq c_{1} d$ for some $c_{1}$ depending only on $\Omega$.

Construction of the set $\left\{P_{1}, \ldots, P_{M-1}\right\}$ is elementary but tedious, so we omit it. 
Next we define a smooth reference domain which we shall use when proving estimates near flat portions of $\partial \Omega$. Let $\tilde{C}=\frac{\sqrt{3}}{2}$, and fix $\tilde{c}$ such that $\frac{1}{2}<\tilde{c}<\tilde{C}$. We then let $P_{0}$ be a domain such that $\partial P_{0}$ is smooth, $P_{0}$ contains the half-ball $B_{\tilde{c}}(0) \cap \mathbb{R}_{+}^{n}$, and $P_{0}$ is contained in the half-ball $B_{\tilde{C}}(0) \cap \mathbb{R}_{+}^{n}$. Also note that $\operatorname{dist}\left(x, \partial P_{0} \backslash\left\{\left(x_{1}, \ldots, x_{n}\right): x_{n}=0\right\}\right)>c_{2}$, where $x \in \mathbb{R}^{n}$ satisfies $x_{i}=0,1 \leq i \leq$ $n-1$, and $0<x_{n} \leq \frac{1}{2}$.

Finally, for notational convenience we let $P_{M}=B_{1 / 2}(0)$.

2.3. Regularized $\delta$-distribution. Our proofs make use of regularized Green's and $\delta$-functions. Here we give properties of regularized (or discrete) $\delta$-functions. To a given point $x_{0} \in \bar{T}$ and parameter $\rho \leq h_{T}$, we first associate a shape-regular simplex $T_{0} \subset T$ such that $x_{0} \in \overline{T_{0}}$ and such that $\left|T_{0}\right|^{1 / n}$ is equivalent to $\rho$. Following for example [SW95], we may then define a function $\delta_{x_{0}} \in C_{0}^{\infty}\left(T_{0}\right)$ such that for any polynomial $P$ of degree less than $r-1$,

$$
P\left(x_{0}\right)=\int_{T_{0}} \delta_{x_{0}} P \mathrm{~d} x
$$

and

$$
\left\|\delta_{x_{0}}\right\|_{W_{k}^{p}\left(T_{0}\right)} \leq C \rho^{-k-n\left(1-\frac{1}{p}\right)}, 1 \leq p \leq \infty, k=0,1 .
$$

In the following proposition, we show that $\delta_{x_{0}}$ behaves like the usual $\delta$ distribution up to a penalty term.

Proposition 2.3. Assume that $u \in C^{k, \alpha}\left(\overline{T_{0}}\right)$ for some $1 \leq k \leq r-1$ and $0<\alpha \leq 1$, and that $\chi \in S_{h}^{r}$. Then there exists a unit vector $\nu$ such that

$$
\left|\nabla(u-\chi)\left(x_{0}\right)\right| \leq C\left(\left|\left(u-\chi, \partial \delta_{x_{0}}\right)\right|+\rho^{k+\alpha-1}|u|_{C^{k, \alpha}\left(\overline{T_{0}}\right)}\right),
$$

where the first-order directional derivative $\partial$ is defined by $\partial v=\nabla v \cdot \nu$.

Proof. First note that for some unit vector $\nu,\left|\nabla(u-\chi)\left(x_{0}\right)\right|=\left|\partial\left(u-u_{h}\right)\left(x_{0}\right)\right|$. Let $T_{0}$ be as described above. For any polynomial $P$ of degree $r-1$, we may then use (2.4) and (2.5) to compute

$$
\begin{aligned}
\partial(u-\chi)\left(x_{0}\right) & =\partial(u-P)\left(x_{0}\right)+\partial(P-\chi)\left(x_{0}\right) \\
& \leq\|\partial(u-P)\|_{L_{\infty}\left(T_{0}\right)}+\left|\int_{T_{0}} \partial(P-\chi) \delta_{x_{0}} \mathrm{~d} x\right| \\
& \leq\|\partial(u-P)\|_{L_{\infty}\left(T_{0}\right)}+\left|\left(\partial(P-u), \delta_{x_{0}}\right)\right|+\left|\left(u-\chi, \partial \delta_{x_{0}}\right)\right| \\
& \leq C\|\partial(u-P)\|_{L_{\infty}\left(T_{0}\right)}+\left|\left(u-\chi, \partial \delta_{x_{0}}\right)\right| .
\end{aligned}
$$

Letting $P$ be an appropriate interpolant of $u$ on the subelement $T_{0}$, we find that

$$
\|\partial(u-P)\|_{L_{\infty}\left(T_{0}\right)} \leq C \rho^{k+\alpha-1}|u|_{C^{k, \alpha}\left(\overline{T_{0}}\right)} .
$$

Collecting (2.7) and (2.8) completes the proof of (2.6). 
2.4. Pointwise estimates for derivatives of Green's functions. Our proofs involve scaling local dual problems to the reference domains $P_{0}, \ldots, P_{M}$. Recall from $\S 2.2$ that for $d \leq d_{0}$, we associate to a given point $x \in \Omega$ a subdomain $P_{x, d} \subset \Omega$. Here $P_{x, d}$ is a copy of some $P_{j}$ that is scaled by $d$, rotated by a rotation matrix $R$, and translated to the point $x$. We must also transform the coefficient matrix $A$ from $P_{x, d}$ to $P_{j}$, which we accomplish via the definition $\tilde{A}(\tilde{x})=A\left(x+d R\left(\tilde{x}-\tilde{x}_{0}\right)\right)$. Here $\tilde{x} \in P_{j}, d \leq d_{0}$ is a parameter as in $\S 2.2, R$ is a rotation matrix, and $\tilde{x}_{0}$ is an appropriately chosen fixed point in $P_{j}$. Finally we note that if $A \in W_{\infty}^{k}\left(P_{x, d}\right)$, then

$$
\|\tilde{A}\|_{W_{\infty}^{k}\left(P_{j}\right)} \leq C\left(d_{0}\right)\|A\|_{W_{\infty}^{k}\left(P_{x, d}\right)} .
$$

In addition, $\tilde{A}$ has the same ellipticity properties as $A$. Also, $C\left(d_{0}\right)$ increases with $d_{0}$, so we may replace $C\left(d_{0}\right)$ with $C(\operatorname{diam}(\Omega))$.

Next we let $G(y, z)$ be a Green's function satisfying $\int_{P_{j}} \tilde{A} \nabla G(y, z) \cdot \nabla v(z) \mathrm{d} z=$ $v(y)$ for sufficiently smooth $v \in H_{0}^{1}\left(P_{j}\right)$. The following pointwise estimates for derivatives of $G$ in the case that $P_{j}$ is smooth or convex are essential to our proofs.

Lemma 2.4. Assume that $\tilde{A}$ and $\partial P_{j}$ are sufficiently smooth. Then for $|\alpha+\beta|>0$,

$$
\left|D_{y}^{\alpha} D_{z}^{\beta} G(y, z)\right| \leq C_{G}|y-z|^{2-n-|\alpha+\beta|} .
$$

Here $C_{G}$ depends on $P_{j}$ and the ellipticity properties and sufficiently strong Sobolev norms of $\tilde{A}$. Using (2.9) and the following comments, we may restate this dependence as: $C_{G}$ depends on the diameter of $\Omega$ and on the ellipticity properties and sufficiently strong Sobolev norms of $A$ on $P_{x, d}$.

Assume that $\tilde{A}$ is Dini-continuous, $P_{j}$ is a convex polyhedron, and $|\beta| \leq 1$ and $|\alpha| \leq 1$. Then (2.10) holds if $n>2$, and for $n=2$,

$$
\left|D_{y}^{\alpha} D_{z}^{\beta} G(y, z)\right| \leq C_{G}|y-z|^{2-n-|\alpha+\beta|} \log \frac{1}{|y-z|} .
$$

Here $C_{G}$ depends on $d_{0}$, the ellipticity properties and Dini-continuity of $A$ on $P_{x, j}$, and the geometry of $P_{j}$.

The estimate (2.10) may be found in [Kr69] for smooth domains and coefficients and for arbitrary $\alpha$ and $\beta$. For $|\alpha|,|\beta| \leq 1$, Dini-continuous $\tilde{A}$, and $n \geq 3$ it may be found in GW82 assuming that $\partial \Omega$ satisfies a uniform exterior sphere condition. This condition is met by both convex and smooth domains. The proof given in GW82 does not carry directly over to $n=2$ due to the logarithmic nature of the singularity, but one may use the same method to obtain the suboptimal estimate (2.11) so long as the estimate

$$
|G(x, y)| \leq C(\lambda, \Lambda, \Omega) \log \frac{1}{|x-y|}
$$

is known. This estimate is contained in [DM95] under the weak restrictions of $L_{\infty}$ and uniformly elliptic coefficients and Lipschitz boundary $\partial \Omega$. The suboptimal estimate (2.11) will only add an additional logarithmic factor to the results of Corollary 3.2 below (for cases where $D$ is close to a convex corner of $\partial \Omega$ ) in the case $n=2$. 


\section{Theoretical Results}

In this section we first prove Theorem 1.1 and Corollary 3.2 and then state and prove a corollary which allows estimation of $\left\|\nabla\left(u-u_{h}\right)\right\|_{L_{\infty}(D)}$ when $D$ is close to convex corners in $\Omega$.

3.1. Proof of Theorem 1.1. We first let $\tilde{\rho}$ be a parameter satisfying $\rho \leq \tilde{\rho} \leq \underline{h}$ which we shall fix later. Let then $\{K\}$ be a finite set of shape-regular simplices whose closures cover $D$, whose diameters are equivelent to $\tilde{\rho}$, and which have the property that for each $K \in\{K\}, K \cap D \neq \emptyset$ and $K \subset T$ for some $T \in \mathcal{T}_{D}$. Let then

$$
\mathcal{K}=\operatorname{interior}\left(\bigcup_{\{K\}} \bar{K}\right)
$$

and note that $D \subset \mathcal{K} \subset D_{\tilde{\rho}}$.

Assume that $\left\|\nabla\left(u-u_{h}\right)\right\|_{L_{\infty}(\mathcal{K})}=\left|\nabla\left(u-u_{h}\right)\left(x_{0}\right)\right|$, and let $T_{0}$ be the simplex of size $\rho$ associated to $x_{1}$ in $\$ 2.3$. Let then $x_{0}$ satisfy

$$
\left\|\nabla\left(u-u_{h}\right)\right\|_{L_{\infty}(\mathcal{K})}=\left|\nabla\left(u-u_{h}\right)\left(x_{0}\right)\right| .
$$

Employing (2.6) with $k=1$ yields

$$
\begin{aligned}
\left\|\nabla\left(u-u_{h}\right)\right\|_{L_{\infty}(D)} & \leq\left\|\nabla\left(u-u_{h}\right)\right\|_{L_{\infty}(\mathcal{K})} \\
& \leq C\left|\left(u-u_{h}, \partial \delta_{x_{0}}\right)\right|+C \rho^{\alpha}|u|_{C^{1, \alpha}(\overline{\mathcal{K}})} .
\end{aligned}
$$

To complete the proof of Theorem 1.1, we let $\tilde{\rho}=\rho$ above and then associate a scaled reference domain to the point $x_{0}$. If $\operatorname{dist}\left(x_{0}, \partial \Omega\right) \geq \frac{d}{2}$, we take $P_{x_{0}, d}=$ $B_{d / 2}\left(x_{0}\right) \subset \Omega$. If $\operatorname{dist}\left(x_{0}, \partial \Omega\right)<\frac{d}{2}$, we associate a scaled copy of $P_{0}$ to $x_{0}$, where $P_{0}$ is defined in $\$ 2.2$. Let $\tilde{x}_{0}$ be the projection of $x_{0}$ onto $\partial \Omega$. Since we have assumed that $\{x \in \partial \Omega: \operatorname{dist}(D, \partial \Omega)<d\}$ is contained in a plane, $B_{\frac{\sqrt{3}}{2} d}\left(\tilde{x}_{0}\right) \cap \Omega$ is a half-ball. Thus we let $P_{x_{0}, d}$ be a copy of $P_{0}$ which is scaled by $d$ and which is translated and rotated so that $x_{0} \in P_{x_{0}, d}$, the flat portion of $P_{x_{0}, d}$ coincides with $\partial \Omega$, and the origin of $P_{0}$ is mapped to $\tilde{x}_{0}$. Note that then $\operatorname{dist}\left(x_{0}, \partial P_{x_{0}, d} \backslash \partial \Omega\right) \geq c_{2} d$ with $c_{2}$ as defined in $\$ 2.2$.

Let $\omega$ be a smooth cutoff function satisfying $0 \leq \omega \leq 1 ; \omega \equiv 1$ on $B_{c_{2} d / 2}\left(x_{0}\right)$; $\omega \equiv 0$ on $\partial B_{3 c_{2} d / 4}\left(x_{0}\right) \backslash \partial \Omega$; and $\left\|D^{j} \omega\right\|_{L_{\infty}\left(P_{x_{0}, d}\right)} \leq C d^{-j}, j \geq 0$. We next define a regularized Green's function $g^{x_{0}}$ which solves a dual problem whose right-hand side is a regularized $\delta$-distribution as defined in $₫ 2.3$. We thus let $g^{x_{0}} \in H_{0}^{1}\left(P_{x_{0}, d}\right)$ solve

$$
\int_{P_{x_{0}, d}} A \nabla v \cdot \nabla g^{x_{0}} \mathrm{~d} x=\int_{P_{x_{0}, d}} v \partial \delta_{x_{0}} \mathrm{~d} x \forall v \in H_{0}^{1}\left(P_{x_{0}, d}\right),
$$

and then compute

$$
\begin{aligned}
\left(u-u_{h}, \partial \delta_{x_{0}}\right)=\left(\omega\left(u-u_{h}\right), \partial \delta_{x_{0}}\right) & \\
= & \int_{P_{x_{0}, d}} A \nabla\left(\omega\left(u-u_{h}\right)\right) \cdot \nabla g^{x_{0}} \mathrm{~d} x \\
= & {\left[\int_{P_{x_{0}, d}} A \nabla\left(u-u_{h}\right) \cdot \nabla\left(\omega g^{x_{0}}\right) \mathrm{d} x\right] } \\
& +\left[\int_{P_{x_{0}, d}}\left(u-u_{h}\right) A \nabla \omega \cdot \nabla g^{x_{0}} \mathrm{~d} x-\int_{P_{x_{0}, d}} g^{x_{0}} A \nabla\left(u-u_{h}\right) \cdot \nabla \omega \mathrm{d} x\right] \\
= & {[I]+[I I] . }
\end{aligned}
$$


First we consider the term $I I$. Integrating by parts, we compute that

$$
-\int_{P_{x_{0}, d}} g^{x_{0}} A \nabla\left(u-u_{h}\right) \cdot \nabla \omega \mathrm{d} x=\int_{P_{x_{0}, d}}\left(u-u_{h}\right) \operatorname{div}\left(g^{x_{0}} A \nabla \omega\right) \mathrm{d} x
$$

so that with $\frac{1}{q}=1-\frac{1}{p}$,

$$
|I I| \leq\left\|u-u_{h}\right\|_{W_{p}^{-k}\left(P_{\left.x_{0}, d\right)}\right.}\left\|A \nabla \omega \cdot \nabla g^{x_{0}}+\operatorname{div}\left(g^{x_{0}} A \nabla \omega\right)\right\|_{W_{q}^{k}\left(P_{x_{0}, d}\right)} .
$$

Applying Hölder's inequality and Leibniz's rule, we find that

$$
\begin{aligned}
\| A \nabla \omega & \cdot \nabla g^{x_{0}}+\operatorname{div}\left(g^{x_{0}} A \nabla \omega\right) \|_{W_{q}^{k}\left(D_{d}\right)} \\
\leq & C\|A\|_{W_{\infty}^{k+1}\left(D_{d}\right)} d^{\frac{n}{q}} \sum_{i+j \leq k}\left[\left|g^{x_{0}}\right|_{W_{\infty}^{i+1}(\operatorname{supp}(\nabla \omega))}|\omega|_{W_{\infty}^{j+1}(\operatorname{supp}(\nabla \omega))}\right. \\
& \left.+\left|g^{x_{0}}\right|_{W_{\infty}^{i}(\operatorname{supp}(\nabla \omega))}|\omega|_{W_{\infty}^{j+2}(\operatorname{supp}(\nabla \omega))}\right] \\
\leq & C\|A\|_{W_{\infty}^{k+1}\left(D_{d}\right)} d^{\frac{n}{q}} \sum_{i+j \leq k}\left[d^{-j-1}\left|g^{x_{0}}\right|_{W_{\infty}^{i+1}(\operatorname{supp}(\nabla \omega))}\right. \\
& \left.+d^{-j-2}\left|g^{x_{0}}\right|_{W_{\infty}^{i}(\operatorname{supp}(\nabla \omega))}\right] .
\end{aligned}
$$

In order to bound $\left|g^{x_{0}}\right|_{W_{\infty}^{i}(\operatorname{supp}(\nabla \omega))}$, we scale to the reference domain $P_{0}$ or $P_{M}$ as appropriate. With $\tilde{x} \in P_{i}(i=0$ or $i=M)$ representing the natural transformation of $x \in P_{x_{0}, d}$ to $P_{i}$, we define $\tilde{g}(\tilde{x})=g^{x_{0}}(x), \tilde{\delta}(\tilde{x})=\delta_{x_{0}}(x)$, and $\tilde{A}(\tilde{x})=A(x)$. It is easy to compute that $-\operatorname{div}(\tilde{A} \nabla \tilde{g})=d \partial \tilde{\delta}$ and $\|\tilde{\delta}\|_{L_{1}\left(P_{j}\right)} \leq C d^{-n}$. Recall that $\omega \equiv 1$ on $B_{c_{2} d / 2}\left(x_{0}\right)$, so that $\operatorname{dist}\left(\operatorname{supp}(\nabla \omega), \operatorname{supp}\left(\delta_{x_{0}}\right)\right) \geq \frac{c_{2} d}{4}$ (since $\left.\rho<d / 8\right)$, and for any $x \in \operatorname{supp}(\nabla \omega), \operatorname{dist}(\tilde{x}, \operatorname{supp}(\tilde{\delta})) \geq \frac{c_{2}}{4}$. Using these facts and applying (2.10), we find that for any $x \in \operatorname{supp}(\nabla \omega)$ and $m \geq 0$,

$$
\begin{aligned}
\left|D^{m} g^{x_{0}}(x)\right| & =d^{-m}\left|D^{m} \tilde{g}(\tilde{x})\right|=d^{-m}\left|\int_{P_{i}} D_{\tilde{x}}^{m} G(\tilde{x}, y) d \partial \tilde{\delta} \mathrm{d} y\right| \\
& =d^{-m+1}\left|\int_{P_{i}} \partial_{y} D_{\tilde{x}}^{m} G(\tilde{x}, y) \tilde{\delta} \mathrm{d} y\right| \\
& \leq d^{-m+1}\left\|\partial D_{x}^{m}(x, \cdot)\right\|_{L_{\infty}\left(\left\{y:|y-\tilde{x}|>.25 c_{2}\right\}\right)}\|\tilde{\delta}\|_{L_{1}\left(P_{i}\right)} \\
& \leq C\left(A, c_{2}, m\right) d^{-m+1-n} .
\end{aligned}
$$

Thus

$$
\begin{aligned}
C\|A\|_{W_{\infty}^{k+1}\left(D_{d}\right)} d^{\frac{n}{q}} & \sum_{i+j \leq k}\left[d^{-j-1}\left|g^{x_{0}}\right|_{W_{\infty}^{i+1}(\operatorname{supp}(\nabla \omega))}\right. \\
& \left.+d^{j+2}\left|g^{x_{0}}\right|_{W_{\infty}^{i}(\operatorname{supp}(\nabla \omega))}\right] \\
\leq & C_{G}\|A\|_{W_{\infty}^{k+1}\left(D_{d}\right)} d^{n-\frac{n}{p}} \sum_{i+j \leq k} d^{-j-1-i-n} \\
\leq & C d^{-1-k-\frac{n}{p}} .
\end{aligned}
$$

Inserting (3.8) into (3.6) and the resulting inequality into (3.5) bounds the term $I I$ as desired.

In order to bound the term $I$, we first note that the definition of $I_{h}$ given in $\$ 2.1$ yields that for $T \in \mathcal{T}_{P_{x_{0}, d}}, \operatorname{supp}\left(I_{h}\left(\omega g^{x_{0}}\right)\right) \subset P_{x_{0}, d}$. Thus we may use (2.1), (2.2), 
and (2.3) and compute as in (4.5) and (4.6) of [De to find that

$$
\begin{aligned}
I= & \int_{P_{x_{0}, d}} A \nabla\left(u-u_{h}\right) \cdot \nabla\left(\omega g^{x_{0}}\right) \mathrm{d} x \\
= & \int_{P_{x_{0}, d}} A \nabla\left(u-u_{h}\right) \cdot \nabla\left(\omega g^{x_{0}}-I_{h}\left(\omega g^{x_{0}}\right)\right) \mathrm{d} x \\
\leq & \sum_{T \subset \mathcal{T}_{P_{x_{0}}, d}} \mathcal{E}_{T}\left(h_{T}^{-1}\left\|\omega g^{x_{0}}-I_{h}\left(\omega g^{x_{0}}\right)\right\|_{L_{1}(T)}+\| \nabla\left(\omega g^{x_{0}}-I_{h}\left(\omega g^{x_{0}}\right) \|_{L_{1}(T)}\right)\right. \\
\leq & \sum_{T \in \mathcal{T}_{P_{T_{x_{0}}}}: T \subset P_{T_{x_{0}}}^{\prime \prime}} \mathcal{E}_{T}\left\|\omega g^{x_{0}}\right\|_{W_{1}^{1}\left(P_{T}\right)} \\
& +\sum_{T \in \mathcal{T}_{P_{d, x_{0}}} \backslash P_{T_{x_{0}}^{\prime \prime}}} h_{T}^{r-1} \mathcal{E}_{T}\left|\omega g^{x_{0}}\right|_{W_{1}^{r}\left(P_{T}\right)} \\
\leq & \mathcal{E}_{T \in \mathcal{T}_{P_{T_{x_{0}}}}: T \subset P_{T_{x_{0}}}^{\prime \prime}}\left\|\omega g^{x_{0}}\right\|_{W_{1}^{1}\left(P_{T}\right)} \\
& +\sum_{T \in \mathcal{T}_{P_{P_{j}, x_{0}}} \backslash P_{T_{x_{0}}^{\prime \prime}}}\left(\frac{h_{T}}{\operatorname{dist}\left(x_{0}, T\right)+h_{T}}\right)^{r-1}\left(\operatorname{dist}\left(x_{0}, T\right)+h_{T}\right)^{r-1}\left|\omega g^{x_{0}}\right|_{W_{1}^{r}\left(P_{T}\right)} \\
\leq & C\left(\left\|\omega g^{x_{0}}\right\|_{W_{1}^{1}\left(P_{x_{0}, d}\right)}+\int_{P_{x_{0}, d} \backslash P_{T_{x_{1}}}^{\prime}}\left|x-x_{0}\right|^{r-1}\left|D^{r}\left(\omega g^{x_{0}}\right)\right| \mathrm{d} x\right) \\
& \cdot \max _{T \in \mathcal{T}_{P_{x_{0}}, d}}\left(\sigma_{x_{0}}(T)\right)^{r-1} \mathcal{E}_{T} .
\end{aligned}
$$

We may compute exactly as in (3.7) (with $\left|x-x_{0}\right|$ replacing d) that

$$
\left|D^{i} g^{x_{0}}(x)\right| \leq C\left|x-x_{0}\right|^{-i+1-n}
$$

for $x \in P_{x_{0}, d} \backslash P_{T_{x_{0}}}^{\prime}$. Thus switching to polar coordinates and noting that $\left|D^{i} \omega\right| \leq$ $d^{-i} \leq t^{-i}$ for $0 \leq t \leq d$, we find that

$$
\begin{aligned}
\int_{P_{x_{0}, d} \backslash P_{T_{x_{1}}}^{\prime}} & \left|x-x_{0}\right|^{r-1}\left|D^{r}\left(\omega g^{x_{0}}\right)\right| \mathrm{d} x \\
& \leq C \int_{C h_{T}}^{d}|t|^{r-1}|t|^{-r+1-n}|t|^{n-1} \mathrm{~d} t \\
& \leq C \int_{C h_{T}}^{d} t^{-1} \leq C \ln \frac{d}{C h_{T}} \leq \ell_{\rho, d} .
\end{aligned}
$$

Using the Poincaré inequality $\left\|g^{x_{0}}\right\|_{L_{1}\left(P_{x_{0}, d}\right)} \leq C d\left\|\nabla g^{x_{0}}\right\|_{L_{1}\left(P_{x_{0}, d}\right)}$ and recalling that $\|\nabla \omega\|_{L_{\infty}\left(P_{x_{0}, d}\right)} \leq C d^{-1}$, we find that $\left\|\omega g^{x_{0}}\right\|_{W_{1}^{1}\left(P_{d, x_{0}}\right)} \leq C\left\|\nabla g^{x_{0}}\right\|_{L_{1}\left(P_{x_{0}, d}\right)}$. One may scale the argument of Lemma 3.8 of De to the reference domain $P_{j}$ associated to $P_{x_{0}, d}$ to find

$$
\left\|\nabla g^{x_{0}}\right\|_{L_{1}\left(P_{x_{0}, d}\right)} \leq C \ell_{\rho, d}
$$

Inserting (3.10) and (3.11) into (3.9) bounds the term $I$ as desired. In order to complete the proof of Theorem (1.1), we note that since $x_{0} \in D^{h}$ and $P_{x_{0}, d} \subset D_{d}$, $\sigma_{x_{0}} \leq \sigma_{D^{h}}=\sigma_{D}$ and $\left\|u-u_{h}\right\|_{W_{p}^{-k}\left(P_{x_{0}, d}\right)} \leq\left\|u-u_{h}\right\|_{W_{p}^{-k}\left(D_{d}\right)}$. 
Remark 3.1. It is possible to prove Theorem 1.1 for $p \geq 2$ under the assumption that solutions $u$ of (1.1) satisfy the regularity shift estimate

$$
\|u\|_{H^{m+2}(\Omega)} \leq C\|f\|_{H^{m}(\Omega)}
$$

for $m=\max (r-1, k)$. The proof of Theorem 4.1 of De relies on such a regularity estimate with $k=0$ to prove that the correct order of weight is obtained.

3.2. Proof of Corollary 1.2. We prove Corollary 1.2 by making appropriate modifications to the proof of Theorem 1.1. First we fix

$$
\tilde{\rho}=\min \left(\eta, \tilde{\eta}, \underline{h}, \frac{C^{*}}{2\left(C^{* *}+C|u|_{C^{1, \alpha}\left(\overline{D_{\tilde{\eta}}}\right)}\right)}, \frac{d}{8}\right) .
$$

We then recall the definition of $\mathcal{K}$ from (3.1) and also recall that $\{K\}$ is composed of elements of size $\tilde{\rho}$. Next note that $x_{1} \in \overline{K_{1}}$ for some $K_{1} \in\{K\}$, where $x_{1}$ is the point specified in the statement of Corollary 1.2. Noting that $K_{1} \subset B_{\nu}\left(x_{1}\right)$ since $\tilde{\rho} \leq \nu$, we then employ Lemma 2.2 to obtain

$$
\begin{aligned}
\left\|\nabla\left(u-u_{h}\right)\right\|_{L_{\infty}(\mathcal{K})} & \geq\left\|\nabla\left(u-u_{h}\right)\right\|_{L_{\infty}\left(K_{1}\right)} \\
& \geq C \tilde{\rho}^{r-1}\left(|u|_{W_{\infty}^{r}\left(K_{1}\right)}-\tilde{\rho}\|u\|_{W_{\infty}^{r+1}\left(K_{1}\right)}\right) \\
& \geq \tilde{\rho}^{r-1}\left(C^{*}-\tilde{\rho} C^{* *}\right) .
\end{aligned}
$$

Here we have incorporated the inconsequential constant $C$ from (3.14) into $C^{*}$ and $C^{* *}$. Since $\tilde{\rho} \leq \frac{C^{*}}{2 C^{* *}}$, we thus have

$$
\left\|\nabla\left(u-u_{h}\right)\right\|_{L_{\infty}(\mathcal{K})} \geq \frac{C^{*}}{2} \tilde{\rho}^{r-1} .
$$

Note next that since $\tilde{\rho} \leq \tilde{\eta}, \mathcal{K} \subset D_{\tilde{\eta}}$ and $\tilde{\rho} \leq \frac{C^{*}}{2 C|u|_{C^{1, \alpha}}(\overline{\mathcal{K}})}$. Defining

$$
\rho=\tilde{\rho}^{\frac{r}{\alpha}}
$$

we find that

$$
\begin{aligned}
C \rho^{\alpha}|u|_{C^{1, \alpha}(\overline{\mathcal{K}})} & \leq \frac{C^{*}}{2^{r}} \tilde{\rho}^{r-1} \\
& \leq \frac{1}{2} \frac{C^{*}}{2} \tilde{\rho}^{r-1} \leq \frac{1}{2}\left\|\nabla\left(u-u_{h}\right)\right\|_{L_{\infty}(\mathcal{K})} .
\end{aligned}
$$

Inserting (3.17) into (3.2) and kicking backing the term $\frac{1}{2}\left\|\nabla\left(u-u_{h}\right)\right\|_{L_{\infty}(\mathcal{K})}$ yields

$$
\left\|\nabla\left(u-u_{h}\right)\right\|_{L_{\infty}(D)} \leq C\left|\left(u-u_{h}, \partial \delta_{x_{0}}\right)\right| .
$$

Bounding $\left|\left(u-u_{h}, \partial \delta_{x_{0}}\right)\right|$ exactly as in the proof of Theorem 1.1 above yields

$$
\left\|\nabla\left(u-u_{h}\right)\right\|_{L_{\infty}(D)} \leq C \ln \frac{d}{\rho} \max _{T \in \mathcal{T}_{D_{d}}} \sigma_{D}(T)^{r-1}+C d^{-1-k-\frac{n}{p}}\left\|u-u_{h}\right\|_{W_{p}^{-k}\left(D_{d}\right)} .
$$

Recalling (3.13) and (3.16) then yields

$$
\begin{aligned}
\left\|\nabla\left(u-u_{h}\right)\right\|_{L_{\infty}(D)} \leq & C_{\text {app }}(r) \tilde{\tilde{\ell}}_{\underline{h}, d} \max _{T \in \mathcal{T}_{D_{d}}} \sigma_{D}(T)^{r-1} \mathcal{E}_{T} \\
& +C_{\mathrm{pol}} d^{-1-k-\frac{n}{p}}\left\|u-u_{h}\right\|_{W_{p}^{-k}\left(D_{d}\right)} .
\end{aligned}
$$

Here

$$
\tilde{\tilde{\ell}}_{\underline{\underline{h}}, d}=\left|\ln \left[\min \left(\frac{1}{d}\left(\frac{C^{*}}{C^{* *}+C|u|_{C^{1, \alpha}(\overline{\mathcal{K}})}}\right)^{\frac{r}{\alpha}}, \frac{\underline{h}^{\frac{r}{\alpha}}}{d}, \frac{\eta^{\frac{r}{\alpha}}}{d}, \frac{\tilde{\eta}^{\frac{r}{\alpha}}}{d}\right)\right]\right| .
$$


Finally we employ the local regularity result

$$
|u|_{C^{1, \alpha}(\bar{K})} \leq C(D, d)\|f\|_{L_{\infty}(\Omega)} .
$$

This result may be obtained by noting that $|u|_{C^{1, \alpha}(\bar{K})} \leq|u|_{C^{1, \alpha}\left(\overline{D_{d / 2}}\right)}$ and applying for example Corollary 8.36 and Theorem 8.15 of [GT98. Inserting (3.19) into (3.18) completes the proof of Corollary 1.2 .

3.3. Results near convex corners of $\Omega$. When $D$ lies near a convex vertex or edge of $\partial \Omega$, it is still possible to prove a weighted a posteriori estimate for $\left\|\nabla\left(u-u_{h}\right)\right\|_{L_{\infty}(D)}$. However, in general we can only show that the local residual portion of the estimate admits a factor of $\sigma_{D}(T)$ and not $\sigma_{D}(T)^{r-1}$ as in (1.5) and (1.7). This is due to the reduced regularity of solutions to elliptic problems on domains which are merely convex and not smooth. In particular, increasing the allowed power of the weight $\sigma_{D}(T)$ requires bounding increasingly high derivatives of the regularized Green's function $g^{x_{0}}$ defined in $\$ 3.1$. For convex domains, we generally only have bounds for second derivatives of solutions to elliptic problems, which corresponds to one factor of $\sigma_{D}(T)$.

Corollary 3.2. Let $\Omega$ be a polygonal or polyhedral domain in $\mathbb{R}^{2}$ or $\mathbb{R}^{3}$. Assume that $D \subset \Omega$ and $0<d \leq d_{0}$ (where $d_{0}$ depends on $\Omega$ ) are such that for each $x \in \partial \Omega$ with $B_{d}(x) \cap D \neq \emptyset$, any component of $B_{d}(x) \cap \Omega$ which has nontrivial intersection with $D$ is convex. Assume also that $A$ is sufficiently smooth and that $u \in C^{1, \alpha}\left(\overline{D_{\tilde{\eta}}}\right)$ for some $\tilde{\eta}>0$ and $0<\alpha<1$. Let $\rho \leq \min \left(\underline{h}, \frac{d}{8}, \tilde{\eta}\right)$. Then for $1 \leq p \leq \infty$,

$$
\begin{aligned}
\left\|\nabla\left(u-u_{h}\right)\right\|_{L_{\infty}(D)} \leq & C_{\text {app }}(r)\left(\ell_{\rho, d}\right)^{\mu(n)} \max _{T \in \mathcal{T}_{D_{d}}} \sigma_{D}(T) \mathcal{E}_{T} \\
& +C_{\mathrm{pol}} d^{-1-\frac{n}{p}}\left\|u-u_{h}\right\|_{L_{p}\left(D_{d}\right)}+C \rho^{\alpha}|u|_{C^{1, \alpha}\left(\overline{D_{\rho}}\right)} .
\end{aligned}
$$

Here $\mu(2)=2$ and $\mu(3)=1$. Next, assume in addition that there exist a point $x_{1} \in D$ and an $\eta>0$ such that $\left|D^{\gamma} u\left(x_{1}\right)\right| \geq C^{*}$ for some multiindex $\gamma$ with $|\gamma|=r$ and $\|u\|_{W_{\infty}^{r+1}\left(B_{\eta}\left(x_{1}\right)\right)} \leq C^{* *}$. Then

$$
\begin{aligned}
\left\|\nabla\left(u-u_{h}\right)\right\|_{L_{\infty}(D)} \leq & C_{\text {app }}(r)\left(\hat{\ell}_{\underline{h}, d}\right)^{\mu(n)} \max _{T \in \mathcal{I}_{D_{d}}} \sigma_{D}(T) \mathcal{E}_{T} \\
& +C_{\mathrm{pol}} d^{-1-k-\frac{n}{p}}\left\|u-u_{h}\right\|_{W_{p}^{-k}\left(D_{d}\right)} .
\end{aligned}
$$

Here

$$
\hat{\ell}_{\underline{h}, d}=\left|\ln \left[\min \left(\frac{1}{d}\left(\frac{C^{*}}{C^{* *}+C|u|_{C^{1, \alpha}\left(D_{\tilde{\eta}}\right.}}\right)^{\frac{r}{\alpha}}, \frac{h^{\frac{r}{\alpha}}}{d}, \frac{\eta^{\frac{r}{\alpha}}}{d}, \frac{\tilde{\eta}^{\frac{r}{\alpha}}}{d}\right)\right]\right| .
$$

Proof of Corollary 3.2. The proof of Corollary 3.2 requires only relatively small modifications to the proofs of Theorem 1.1 and Corollary 1.2. For the sake of brevity, we shall thus only redefine notation as necessary and shall refer back to the previous proofs when possible.

First we choose a point $x_{0} \in \mathcal{K}$ as in (3.2). If $\operatorname{dist}\left(x_{0}, \partial \Omega\right)>c_{3} d$, we may proceed exactly as in the proof of Theorem 1.1. If $\operatorname{dist}\left(x_{0}, \partial \Omega\right) \leq c_{3} d$, the hypotheses of Corollary 3.2 imply that the polygon $P_{i}$ and scaled polygon $P_{x_{0}, d}$ associated to $x_{0}$ in property (2) of $\$ 2.2$ are convex. Also note that $P_{x_{0}, d} \subset D_{d}$ since $\operatorname{diam}\left(P_{i}\right) \leq 1$. As in the proof of Theorem 1.1. we let $\omega$ be a smooth cutoff function satisfying $0 \leq \omega \leq 1$, $\omega \equiv 1$ on $B_{\frac{c_{1}}{2} d}\left(x_{0}\right)$ for some $c_{1}$ as defined in $₫ 2.2, \omega \equiv 0$ on $\partial B_{.75 c_{2} d}\left(x_{0}\right) \backslash \partial \Omega$, and $\left\|D^{j} \omega\right\|_{L_{\infty}\left(P_{x_{0}, d}\right)} \leq C d^{-j}, j=0,1,2$. 
Next we define $g^{x_{0}}$ as in (3.3) and then proceed as in (3.4) through (3.8) while recalling that $k=0$. We must be careful that we only apply the Green's function estimates (2.10) and (2.11) with $|\alpha| \leq 1$ an $|\beta| \leq 1$. Indeed, we see that when $k=0$ we only apply (3.7) with $0 \leq i \leq 1$. Thus we may bound the term $I I$ from (3.4) essentially exactly as before. Note also that since we only apply the suboptimal Green's function estimate (2.11) at a unit distance from the singularity, the extra logarithmic factor when $n=2$ does not enter into the estimate of the term $I I$.

Next we bound the term $I$ from (3.7). Computing as in (3.9), we find that

$$
\begin{aligned}
I \leq & \left(\left\|\omega g^{x_{0}}\right\|_{W_{1}^{1}\left(P_{x_{0}, d}\right)}+\int_{P_{x_{0}, d} \backslash P_{T_{x_{0}}}^{\prime}}\left|x-x_{0} \| D^{2}\left(\omega g^{x_{0}}\right)\right| \mathrm{d} x\right) \\
& \cdot \max _{T \in \mathcal{T}_{P_{x_{0}}, d}} \sigma_{x_{0}}(T) \mathcal{E}_{T} .
\end{aligned}
$$

Next note that $\left|x-x_{0}\right| \leq C d$ for $x \in P_{x_{0}, d}$. Using this fact, scaling the argument of Lemma 4.5 of De, and using (3.7), we find that

$$
\begin{aligned}
\int_{P_{x_{0}, d} \backslash P_{T_{x_{0}}}}\left|x-x_{0}\right|\left|D^{2}\left(\omega g^{x_{0}}\right)\right| \mathrm{d} x \leq \int_{P_{x_{0}, d} \backslash P_{T_{x_{0}}}} \omega^{2}\left|x-x_{0}\right|\left|D^{2} g^{x_{0}}\right| \mathrm{d} x \\
\quad+\int_{\operatorname{supp}(\nabla \omega)} d|\nabla \omega|\left|\nabla g^{x_{0}}\right| \mathrm{d} x+\int_{\operatorname{supp}(\nabla \omega)} d\left|D^{2} \omega\right|\left|g^{x_{0}}\right| \mathrm{d} x \\
\leq \int_{P_{x_{0}, d} \backslash P_{T_{x_{0}}}}\left|x-x_{0} \| D^{2} g^{x_{0}}\right| \mathrm{d} x \\
\quad+C d^{n}\left\|\nabla g^{x_{0}}\right\|_{L_{\infty}(\operatorname{supp}(\nabla \omega))}+C d^{n-1}\left\|g^{x_{0}}\right\|_{L_{\infty}(\operatorname{supp}(\nabla \omega))} \\
\leq C\left(\left(\ell_{\rho, d}\right)^{\mu(n)}+d^{n}\left\|\nabla g^{x_{0}}\right\|_{L_{\infty}(\operatorname{supp}(\nabla \omega))}+d^{n-1}\left\|g^{x_{0}}\right\|_{L_{\infty}(\operatorname{supp}(\nabla \omega))}\right) \\
\leq C\left(\left(\ell_{\rho, d}\right)^{\mu(n)}+1\right) .
\end{aligned}
$$

Collecting (3.11) and (3.24) into (3.23) yields

$$
I \leq C\left(\ell_{\rho, d}\right)^{\mu(n)} \max _{T \in \mathcal{T}_{P_{x_{0}}, d}} \sigma_{x_{0}}(T) \mathcal{E}_{T},
$$

which completes the proof of (3.20) when $D=x_{0}$. In order to complete the proof of (3.20) when $D$ is an arbitrary set fulfilling the given hypotheses, we again note that $\sigma_{x_{0}} \leq \sigma_{D^{h}}=\sigma_{D}$ and $\left\|u-u_{h}\right\|_{L_{p}\left(P_{x_{0}, d}\right)} \leq\left\|u-u_{h}\right\|_{L_{p}\left(D_{d}\right)}$.

The proof of (3.21) requires very little modification of the proof of Corollary 1.2, The only difference is that we are not aware of a reference for the local regularity estimate (3.19) in the current situation with $D$ near a convex, but not necessarily smooth, portion of $\partial \Omega$.

\section{Controlling the pollution term}

In this section we discuss strategies for controlling the pollution term $\left\|u-u_{h}\right\|_{W_{p}^{-k}(\Omega)}$ arising in Theorem 1.1. In principle it is most efficient to use the weakest possible norm to measure the pollution error. However, in practice choosing a norm weaker than an $L_{p}$ norm is not always possible, and for computational efficiency choosing the $L_{\infty}$ norm is often sufficient.

First we discuss conditions under which we may measure the pollution error in a negative norm, that is, when we may choose $k>0$ in Theorem 1.1 or Corollary 1.2. 
We shall limit our discussion to the case $p=2$. As is noted in [LN03], conveniently bounding $\left\|u-u_{h}\right\|_{W_{2}^{-k}(\Omega)}$ requires the $H^{k+2}$ regularity estimate

$$
\|v\|_{H^{k+2}(\Omega)} \leq C\|f\|_{H^{k}(\Omega)}
$$

for solutions $v$ of (1.1). This estimate generally requires that $A \in W_{\infty}^{k+1}(\Omega)^{n \times n}$ and that $\partial \Omega$ be of class $C^{k+2}$ (though the latter requirement may sometimes be relaxed; cf [NS74], where it is also shown to hold on squares). If (4.1) holds and $\Omega$ is polyhedral, it is not difficult to show, using standard arguments, that

$$
\left\|u-u_{h}\right\|_{W_{2}^{-k}(\Omega)} \leq C(A)\left(\sum_{T \in \mathcal{T}} \eta_{k, 2}(T)^{2}\right)^{\frac{1}{2}} .
$$

Combining this estimate with (1.7) yields a computable a posteriori estimator for $\left\|\nabla\left(u-u_{h}\right)\right\|_{L_{\infty}(D)}$. Note though that if (4.1) holds and $\Omega$ is a convex polyhedron, then under the conditions of Theorem 1.1 it is also possible to prove the global weighted estimate

$$
\left\|\nabla\left(u-u_{h}\right)\right\|_{L_{\infty}(D)} \leq C \ell_{h} \max _{T \in \mathcal{T}}\left(\sigma_{D}(T)\right)^{k+1} \mathcal{E}_{T}+C \underline{h}^{\alpha \beta}|u|_{C^{1, \alpha}(\bar{\Omega})} ;
$$

cf. Remark 3.1. The estimate (4.3) is essentially as sharp as that obtained by inserting (4.2) into (1.7) and is computationally more convenient, so there generally is little to be gained by using the local estimate (1.7) in situations with high regularity. When $\partial \Omega$ is smooth (which is the most realistic situation when assuming (4.1)), rigorous handling of residual estimators near the boundary has not been investigated either for maximum norm residuals or for negative norm-type residuals. The case $p=2$ and $k=0$ was investigated in DR98.

We consider three options for controlling the pollution term when computing on nonconvex polyhedral domains. In our discussion, we shall assume that the operator under consideration is the Laplacian, as not all results cited here are readily available for other operators.

First we describe the a posteriori estimate for $\left\|u-u_{h}\right\|_{L_{2}(\Omega)}$ proved in LN03. for plane polygonal domains. For simplicity we assume that $\Omega \subset \mathbb{R}^{2}$ is a polygonal domain with exactly one re-entrant corner having vertex $V$ and opening angle $\omega>\pi$. Let $t$ denote the distance to $V$, and define the regularized distance $b(x)=$ $\sqrt{t(x)^{2}+h(x)^{2}}$. Let $\beta=1-\frac{\pi}{\omega}$ and let $W_{2}(T)=\max _{x \in T} b(x)^{-\beta}$. Slightly modifying Lemma 3.3 of [LN03] yields

$$
\left\|u-u_{h}\right\|_{L_{2}(\Omega)}^{2} \leq \tilde{C}_{2}(r) \ell_{h} \sum_{T \in \mathcal{T}} W_{2}(T)^{2} \eta_{0,2}(T)^{2} .
$$

Here $\ell_{h}$ is a generic logarithmic factor having the form $\left(\ln \frac{1}{\underline{h}}\right)^{k}$ for some fixed $k \geq 0$. Combining (4.4) with (1.7) yields

$$
\begin{aligned}
\left\|\nabla\left(u-u_{h}\right)\right\|_{L_{\infty}(D)} \leq & C_{\text {app }}(r) \tilde{\ell}_{\underline{h}, d} \max _{T \in \mathcal{T}_{D_{d}}} \sigma_{D}(T)^{r-1} \mathcal{E}_{T} \\
& +C_{2}(r) d^{-2} \ell_{h}\left(\sum_{T \in \mathcal{T}} W_{2}(T)^{2} \eta_{0,2}(T)^{2}\right)^{\frac{1}{2}},
\end{aligned}
$$

where $C_{2}(r)=C_{p o l} \tilde{C}_{2}$ depends on $A, \Omega$, and $r$. Ignoring logarithmic factors, we thus define the computable error estimator

$$
E_{2}=\frac{1}{2}\left[C_{\text {app }}(r) \max _{T \in \mathcal{T}_{D_{d}}} \sigma_{D}(T)^{r-1}+C_{2}(r) d^{-2}\left(\sum_{T \in \mathcal{T}} \rho_{T}^{2} \eta_{0,2}(T)^{2}\right)^{\frac{1}{2}}\right] .
$$


If more than one re-entrant corner is present, the simple weight $W_{2}(T)$ must be replaced by the maximum over the re-entrant corners of the corresponding weights. Also, it is assumed in LN03 that $\omega<2 \pi$, that is, crack domains are not considered. However, the decomposition of solutions of (1.1) into singular and regular parts used for proving (4.4) also holds on crack domains; see for example Da88, Theorem 14.10. The use of (4.4) for $\omega=2 \pi$ is thus at least heuristically justified.

Precise knowledge of singularities on polyhedral domains is not always available, especially in three space dimensions. In these cases one must seek bounds which do not rely explicitly on domain geometry. Computationally it is simplest to measure the pollution term in $L_{\infty}$. Using the earlier works [Noc95] and [DDP00, it was shown in [NSSV] that

$$
\left\|u-u_{h}\right\|_{L_{\infty}(\Omega)} \leq \tilde{C}_{\infty}(r)\left(\ln \frac{1}{\underline{h}}\right)^{2} \max _{T \in \mathcal{T}} h_{T} \mathcal{E}_{T} .
$$

Inserting (4.5) into (1.7) yields

$$
\begin{aligned}
\left\|\nabla\left(u-u_{h}\right)\right\|_{L_{\infty}(D) \leq} \leq & C_{\text {app }}(r) \tilde{\ell}_{\underline{h}, d} \max _{T \in \mathcal{T}_{D_{d}}} \sigma_{D}(T)^{r-1} \mathcal{E}_{T} \\
& +C_{\mathrm{pol}} \tilde{C}_{\infty}(r) \frac{1}{d} \ell_{h} \max _{T \in \mathcal{T}} h_{T} \mathcal{E}_{T} \\
\leq & C \ell_{h} \max _{T \in \mathcal{T}} \max \left(C_{\mathrm{app}}(r) \tilde{\sigma}_{D}(T)^{r-1}, C_{\infty}(r) \frac{h_{T}}{d}\right) \mathcal{E}_{T} .
\end{aligned}
$$

Here $C_{\infty}(r)$ depends on $A, \Omega$, and $r, \tilde{\sigma}_{D}(T)=\sigma_{D}(T)$ if $T \in \mathcal{T}_{D_{d}}$ and $\tilde{\sigma}(T)=0$ otherwise, and $\ell_{h}$ is again a generic logarithmic factor. We thus define

$$
E_{\infty}=\max _{T \in \mathcal{T}} \max \left(C_{\text {app }}(r) \tilde{\sigma}_{D}(T)^{r-1}, C_{\infty}(r) \frac{h_{T}}{d}\right) \mathcal{E}_{T} .
$$

A third option is to measure the pollution term in $L_{p}$ with $p$ "large" but not $\infty$. For solutions to (1.1) with $A=I$ on arbitrary polyhedral or polygonal domains in $\mathbb{R}^{2}$ or $\mathbb{R}^{3}$,

$$
\|u\|_{W_{q}^{2}(\Omega)} \leq C_{q}\|f\|_{L_{q}(\Omega)}
$$

for $1<q<4 / 3$; cf. Da92. This estimate enables the construction of a residual estimator in $L_{p}$, where $\frac{1}{p}=1-\frac{1}{q}$ and $1<q<4 / 3$. Given (4.6), it is not difficult to show using a standard duality argument that

$$
\left\|u-u_{h}\right\|_{L_{p}(\Omega)} \leq C_{q}(r)\left(\sum_{T \in \mathcal{T}} \eta_{0, p}(T)^{p}\right)^{\frac{1}{p}}
$$

In our computational experiments we shall choose $p=5$, so that $q=\frac{5}{4}$ and $C_{q}$ is unknown but fixed. Inserting (4.7) with $p=5$ into (1.7) yields

$$
\left\|\nabla\left(u-u_{h}\right)\right\|_{L_{\infty}(D)} \leq C_{\text {app }}(r) \ell_{\rho, d} \max _{T \in \mathcal{T}_{D_{d}}} \sigma_{D}(T)^{r-1} \mathcal{E}_{T}+C_{5}(r)\left(\sum_{T \in \mathcal{T}} \eta_{0,5}(T)^{5}\right)^{\frac{1}{5}},
$$

where $C_{5}(r)=C_{q}(r) C_{\text {pol }}$ depends on $A, \Omega$, and $r$. We thus define

$$
E_{5}=\frac{1}{2}\left[C_{\mathrm{app}}(r) \max _{T \in T_{D_{d}}} \sigma_{D}(T)^{r-1} \mathcal{E}_{T}+C_{5}(r)\left(\sum_{T \in \mathcal{T}} \eta_{0,5}(T)^{5}\right)^{\frac{1}{5}}\right] .
$$




\section{Computational Examples}

In this section we describe computational experiments whose purpose is to illustrate Theorem 1.1, Corollary [1.2] and Corollary 3.2 in the case that $\Omega$ is a plane crack domain.

5.1. Algorithm. Our computational algorithm is as follows. We use the standard adaptive procedure of recursively solving the discrete equation (1.2) on an initial mesh, marking the mesh for refinement using the chosen error indicator, refining the mesh, and then repeating the procedure until a given error tolerance is reached. We shall use a "maximum strategy" (see, e.g., Ver94) for deciding which elements to mark for refinement, that is, we mark the element $\tilde{T}$ for refinement if

$$
I(\tilde{T}) \geq \theta \max _{T \in \mathcal{T}} I(T),
$$

where $I$ is the relevant elementwise error indicator and $0<\theta<1$ is chosen appropriately. When measuring the pollution term in $L_{\infty}$, we mark the element $\tilde{T}$ for refinement if

$$
\max \left(C_{\text {app }}(r) \tilde{\sigma}_{D}(\tilde{T})^{r-1}, C_{\infty}(r) \frac{h_{\tilde{T}}}{d}\right) \mathcal{E}_{\tilde{T}} \geq .25 E_{\infty}
$$

When measuring the pollution error in $L_{2}$ or $L_{5}$, a direct comparison between elementwise residuals arising from the pollution and approximation terms is not possible. Instead, we set

$$
e_{\infty}=C_{\mathrm{app}}(r) \max _{T \in \mathcal{T}_{D_{d}}} \sigma_{D}(T)^{r-1} \mathcal{E}_{T}
$$

Letting $W_{5}(T)=1$, we also define for $p=2$ or $p=5$

$$
e_{p}=C_{p}(r)\left(\sum_{T \in \mathcal{T}} W_{p}(T)^{p} \eta_{0, p}(T)^{p}\right)^{\frac{1}{p}},
$$

so that $E_{p}=\frac{1}{2}\left(e_{\infty}+e_{p}\right)$. We employ the following simple algorithm for determining which elements of the mesh to refine:

(1) If $\frac{1}{2} e_{\infty} \geq .4 E_{p}$ :

for each $\tilde{T} \in \mathcal{T}_{D_{d}}$, mark $\tilde{T}$ for refinement if

(2) If $\frac{1}{2} e_{p} \geq .4 E_{p}$ :

$$
C_{\text {app }}(r) \sigma_{D}(\tilde{T})^{r-1} \mathcal{E}_{\tilde{T}} \geq .25 e_{\infty} .
$$

for each $\tilde{T} \in \mathcal{T}$, mark $\tilde{T}$ for refinement if

$$
W_{p}(\tilde{T}) \eta_{0, p}(\tilde{T}) \geq .5 \max _{T \in \mathcal{T}} W_{p}(T) \eta_{0, p}(T) .
$$

Thus we refine the mesh based on the elementwise approximation or pollution error indicators if the corresponding error estimator $e_{\infty}$ or $e_{p}$ accounts for at least $40 \%$ of the total error estimate $E_{p}$. We note that a similar marking strategy for comparing residual contributions which accumulate differently was also used in [NSV03] and NSSV. The threshold values $\theta=.25$ in (5.1) and $\theta=.5$ in (5.2) were chosen based on computational experience and may be varied. Finally, all computations were performed using the finite element toolbox ALBERTA [SS00, SS05].

The constants $C_{\text {app }}(r)$ and $C_{p}(r), p=2,5, \infty$, must also be estimated. The choice of these constants affects the effectiveness of the above algorithm both for error estimation and error indication, that is, in choosing which elements to mark for refinement. In particular, the ratio $\frac{C_{\text {app }}}{C_{p}}$ must be chosen properly in order to 
gain an effective error indicator. For a given subdomain $D$, error estimator $E_{p}$, and discrete solution $u_{h}$, we define the effectivity index

$$
I_{\text {eff }}(D)=\frac{\left\|\nabla\left(u-u_{h}\right)\right\|_{L_{\infty}(D)}}{E_{p}} .
$$

Ideally $I_{\text {eff }}(D)=1$. In our computations $C_{\text {app }}(r)$ was fixed by running simple model problems on square domains and choosing values of $C_{\text {app }}(r)$ for which $I_{\text {eff }}(\Omega) \approx 1$. This procedure yielded $C_{\text {app }}(4)=.13$ (cubics) and $C_{\text {app }}(3)=.26$ (quadratics). $C_{p}(r)$ was fixed in an experiment described below. The resulting values we use are $C_{2}(3)=.08 C_{\text {app }}(3) ; C_{5}(3)=.06 C_{\text {app }}(3) ; C_{\infty}(3)=.05 C_{\text {app }}(3)$; $C_{2}(4)=.06 C_{\mathrm{app}}(4) ; C_{5}(4)=.04 C_{\mathrm{app}}(4) ;$ and $C_{\infty}(4)=.025 C_{\mathrm{app}}(4)$.

5.2. Experiments illustrating Corollary 1.2. Let $\Omega_{1}=(-1,1) \times(-1,1) \backslash$ $[0,1) \times\{0\} . \Omega_{1}$ is depicted in Figure 1, along with the initial mesh used for all computations on $\Omega_{1}$. With $t$ and $\theta$ denoting polar coordinates, let

$$
\phi(t, \theta)=t^{1 / 2} \sin \frac{\theta}{2}
$$

and

$$
u(x, y)=\cos \frac{\pi x}{2} \cos \frac{\pi y}{2} \phi(t(x, y), \theta(x, y)) .
$$

Note that $\left.u\right|_{\partial \Omega}=0$ and that $\phi$ is the (most) singular function which naturally arises in expansions of solutions of (1.1) into regular and singular parts. Additionally, we take $A=I$ and let $f=-\Delta u$.

In our experiments we varied several parameters: the subdomain $D$ under consideration, the polynomial degree $r-1$, and the norm which is used to measure the pollution term. Let $D_{1}=\{(x, y) \in \Omega:|(x, y)-(.05,0)|<0.025, y>0\}$. Note that $D_{1}$ is relatively close to the crack tip $(0,0)$ and lies on one side of the crack, as pictured in Figure 1 When estimating $\left\|\nabla\left(u-u_{h}\right)\right\|_{L_{\infty}\left(D_{1}\right)}$ we shall take $d=0.025$ so that $D_{1, d}=\left\{(x, y) \in \Omega:\left|(x, y)-x_{1}\right|<0.05, y>0\right\}$. Our second subdomain is removed from the crack tip. Let $\left(x_{2}, y_{2}\right)=(0,1)$, and let $D_{2}=\{(x, y) \in \Omega:|(x, y)-(0,1)|<.1\}$. We shall take $d=0.8$ when estimating $\left\|\nabla\left(u-u_{h}\right)\right\|_{L_{\infty}\left(D_{2}\right)}$, so $D_{2, d}$ is a half-disc of radius .9 centered at $x_{2}$.
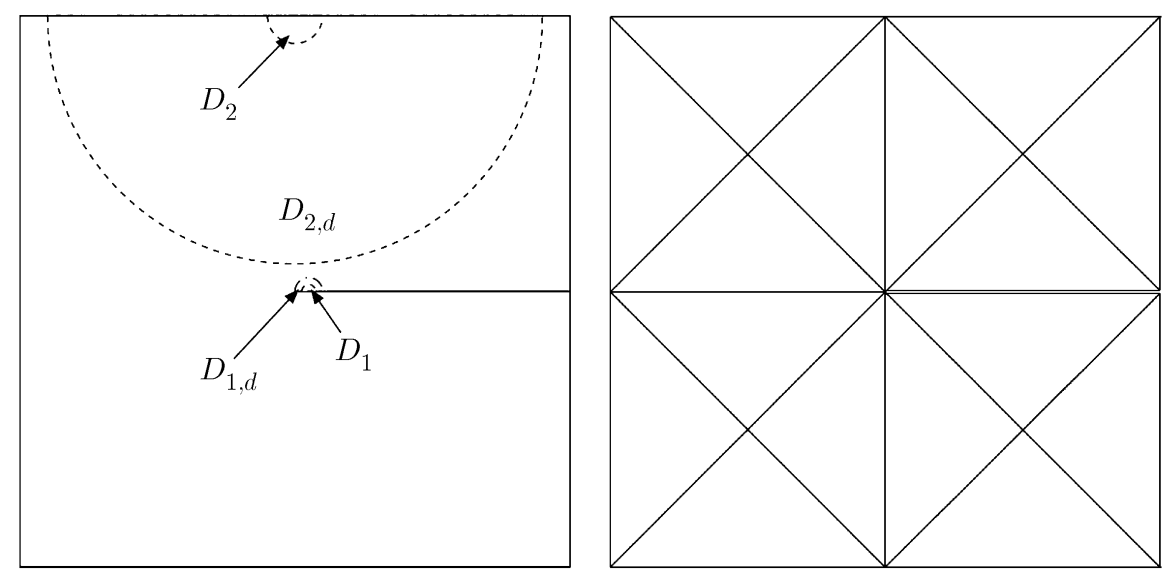

Figure $1 . \Omega_{1}$ along with subdomains $D_{1}, D_{1, d}, D_{2}$, and $D_{2, d}$, and the initial mesh. 
Experiment 1 (Approximation of $\left.\nabla u\right|_{D_{2}}$ ). Computations on the subdomain $D_{2}$ were used to fix the constants $C_{p}(r)$. In particular, we took $C_{p}(r)$ to be the smallest choice of $C_{p}(r)$ for which $I_{\text {eff }}\left(D_{2}\right) \leq 1$ for all iterations of the resulting adaptive algorithm. Note that this is a rather conservative way to choose $C_{p}$ and generally results in overapproximation of the error. Starting with the initial mesh displayed in Figure 1, the algorithm described in the preceding section was employed with a tolerance of $10^{-5}$ for both quadratic $(r=3)$ and cubic $(r=4)$ elements. Using quadratic elements, 93598 degrees of freedom were required to reach the prescribed tolerance using $E_{2}, 88790$ using $E_{5}$, and 112193 using $E_{\infty}$. Using cubic elements, 14487 elements were required to reach the prescribed tolerance using $E_{2}, 13837$ using $E_{5}$, and 17152 using $E_{\infty}$. Thus slightly better results are obtained here by using the $L_{5}$ norm to measure the pollution error, though the performance of algorithms resulting from all three choices of $p$ was competitive. For higher numbers of degrees of freedom, the effectivity index was between about .2 and .6 for all choices of error indicator, confirming that our choice of $C_{p}(r)$ was perhaps too conservative.

Experiment 2 (Approximation of $\left.\nabla u\right|_{D_{1}}$ ). The same algorithm as above was used for computations on $D_{1}$. A graph of the error reduction obtained using $E_{p}$ with quadratic and cubic elements and with $p=2,5, \infty$ is displayed in Figure 2 and Figure 3. Here the best results for both cubic and quadratic elements were obtained using $E_{\infty}$, with similar results being obtained using $E_{5}$ and considerably worse results being obtained using $E_{2}$. Graphs of the effectivity indices resulting from using cubic elements are shown in Figure 4. The effectivity indices for quadratic elements are similar. In particular, for both cubics and quadratics $I_{\text {eff }}\left(D_{1}\right)$ is of moderate size even for small numbers of degrees of freedom, and remains between about .4 and 1.6 for larger numbers of degrees of freedom.

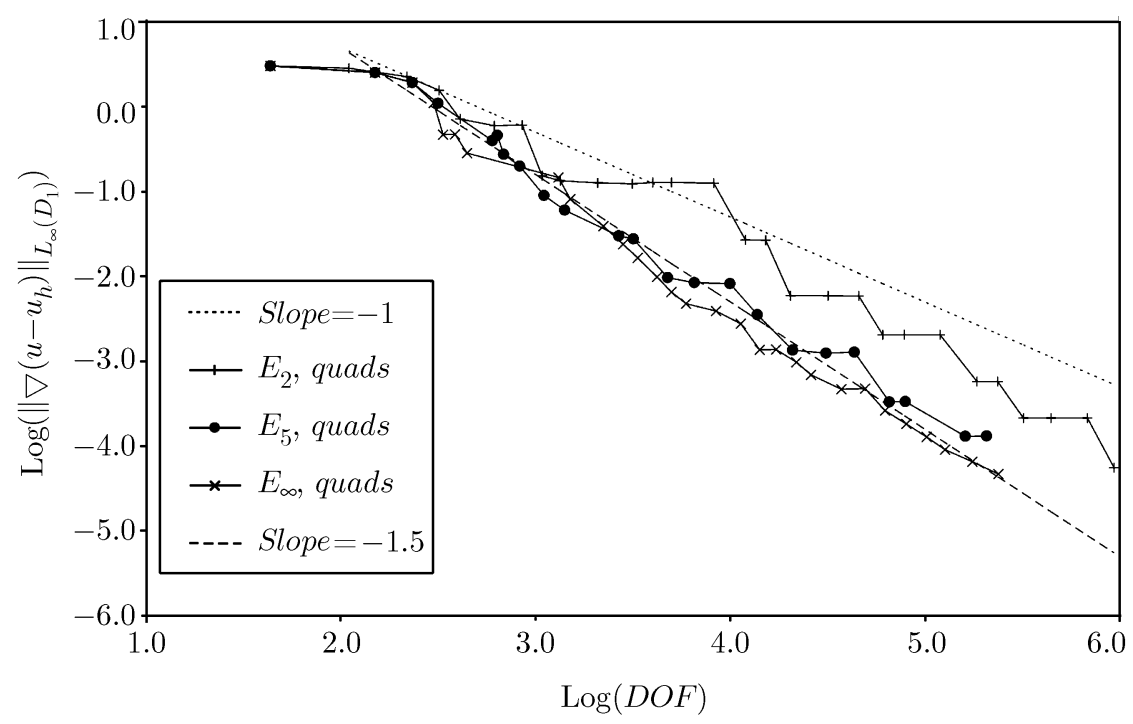

Figure 2. Error reduction when using quadratic elements to approximate $\left.\nabla u\right|_{D_{1}}$. 


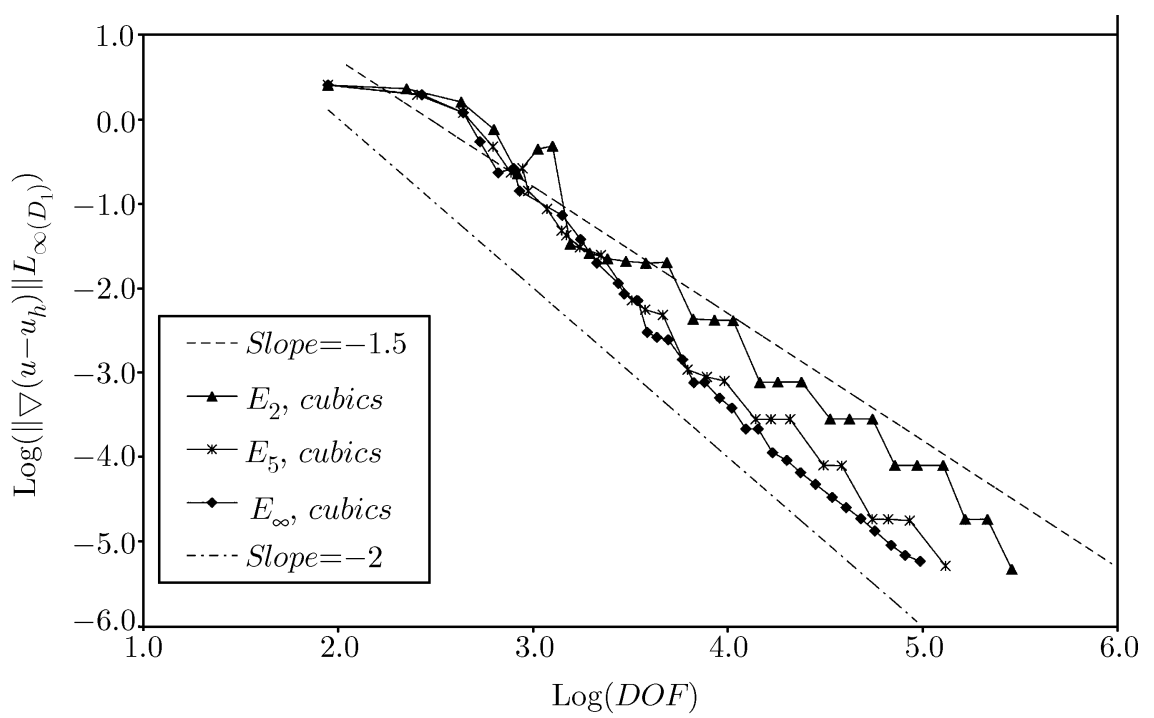

Figure 3. Error reduction when using quadratic and cubic elements to approximate $\left.\nabla u\right|_{D_{1}}$.

We conjecture that the poorer results obtained when using $E_{2}$ are due to overapproximation of the pollution error because of the factor $d^{-1-\frac{n}{p}}$. Here this factor is $d^{-1}=40$ when $p=\infty$ and $d^{-2}=1600$ when $p=2$. Thus the extra regularity gained by approximating the pollution term in the weaker $L_{2}$ norm does not compensate for the increased penalty due to the small size of the domain

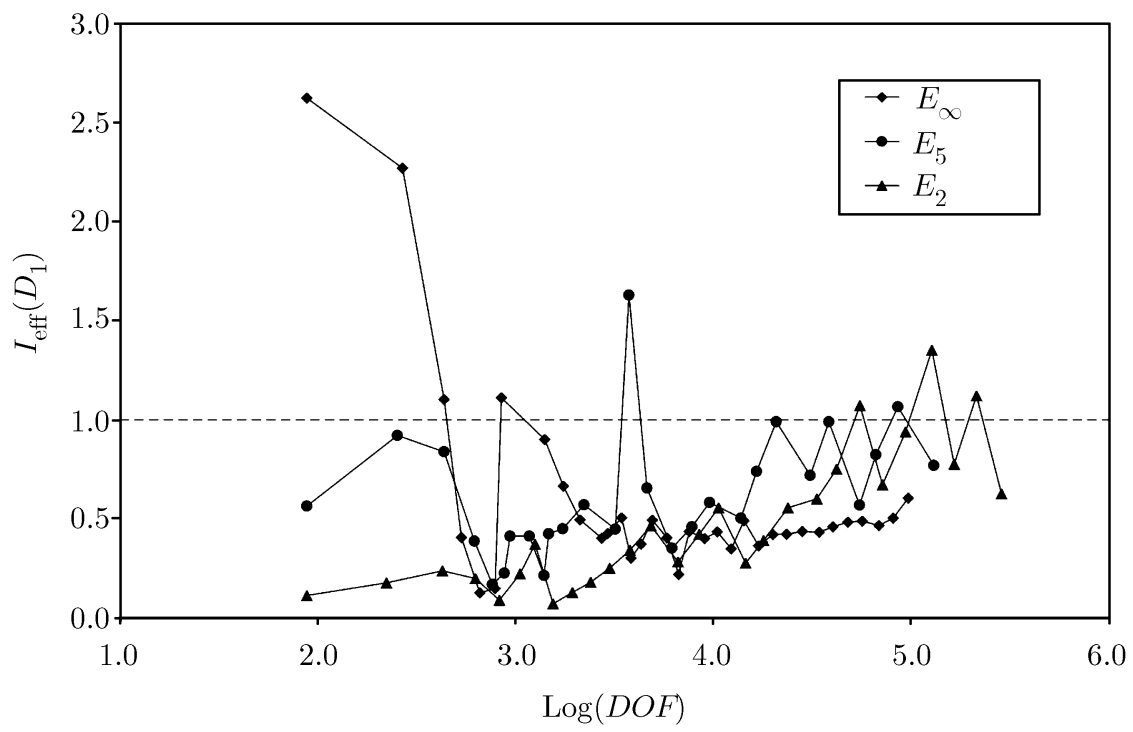

FiguRE 4. Effectivity indices resulting from using cubic elements to approximate $\left.\nabla u\right|_{D_{1}}$. 
$D_{1, d}$. It is interesting to note that on crack domains $\left\|u-u_{h}\right\|_{L_{2}(\Omega)}$ has a strictly higher rate of convergence than $\left\|u-u_{h}\right\|_{L_{\infty}(\Omega)}$ on quasiuniform meshes. This fact might seem to indicate that it is more efficient to measure the pollution term in $L_{2}$ than in $L_{\infty}$. As was shown in SW79, however, the mesh may be refined so that $\left\|u-u_{h}\right\|_{L_{\infty}}$ decreases optimally with the number of degrees of freedom, and numerical tests in [DDP00] confirm that adaptive algorithms based on residual estimators for $\left\|u-u_{h}\right\|_{L_{\infty}(\Omega)}$ lead to such a refined mesh.

Note also that since $D_{1}$ has positive measure, the error should asymptotically decline as $D O F^{-1}$ for quadratic elements and $D O F^{-1.5}$ for cubic elements, where $D O F$ is the number of degrees of freedom. However, in Figures 2 and 3 the error appears to decline as $D O F^{-1.5}$ and $D O F^{-2}$ for quadratic and cubic elements, respectively, at least for $p=5$ and $p=\infty$. These are the optimal rates of convergence for the pollution term when it is measured in $L_{p}, p=2,5, \infty$. It appears that since $\left|D_{1}\right| \ll|\Omega|$, the higher rate of convergence for the pollution term $\left\|u-u_{h}\right\|_{L_{p}(\Omega)}$ dominates in the pre-asymptotic range.

Experiment 3 (Effect of the weight $\sigma_{D}$ ). With our final experiment on $\Omega_{1}$ we illustrate the effect of the weight $\sigma_{D}$. An a posteriori counterpart to the "traditional" unweighted local maximum norm estimates of [SW95] would lead for example to the estimator

$$
\tilde{E}_{\infty}=\max _{T \in \mathcal{T}} \max \left(C_{\text {app }}(r) \delta_{D_{d}}(T), C_{\infty}(r) \frac{h_{T}}{d}\right) \mathcal{E}_{T},
$$

where $\delta_{D_{d}}(T)=1$ if $T \in \mathcal{T}_{D_{d}}$ and $\delta_{D_{d}}(T)=0$ otherwise. Note that when $d=$ .025 the subdomain $D_{1, d}$ abuts directly on the crack tip, where $\nabla u$ is unbounded. Assume that $h_{T_{\text {tip }}} \ll d, T_{\text {tip }} \in \mathcal{T}_{D_{d}}$, and that $\overline{T_{\text {tip }}}$ contains the crack tip. Using the weighted estimator $E_{\infty}$, we see that the local residual contribution from $T_{\text {tip }}$ is

$$
\begin{aligned}
& \max \left(C_{\text {app }}(r) \sigma_{D}\left(T_{\text {tip }}\right)^{r-1}, C_{\infty}(r) \frac{h_{T_{\text {tip }}}}{d}\right) \mathcal{E}_{T_{\text {tip }}} \\
& \quad \leq C \max \left(C_{\text {app }}(r)\left(\frac{h_{T_{\text {tip }}}}{d}\right)^{r-1}, C_{\infty}(r) \frac{h_{T_{\text {tip }}}}{d}\right) \mathcal{E}_{T_{\text {tip }}} \leq C(r) \frac{h_{T_{\text {tip }}}}{d} \mathcal{E}_{T_{\text {tip }}} .
\end{aligned}
$$

That is, the residual contribution from $T_{\text {tip }}$ is comparable to an $L_{\infty}$-type residual estimator as $\mathcal{E}_{T_{\text {tip }}}$ is multiplied by a factor of $h_{T_{\text {tip }}}$. Using $\tilde{E}_{\infty}$ with $d=.025$, on the other hand, yields an elementwise contribution of

$$
\max \left(C_{\text {app }}(r), C_{\infty}(r) \frac{h_{T_{\text {tip }}}}{d}\right) \mathcal{E}_{T_{\text {tip }}} \geq c(r) \mathcal{E}_{T_{\text {tip }}} .
$$

Since $\mathcal{E}_{T_{\text {tip }}}$ heuristically measures the $W_{\infty}^{1}$ error over an area where $\nabla u$ is not bounded, we expect $\mathcal{E}_{T_{\text {tip }}}$ to blow up as the mesh is refined. One may easily confirm this expectation in practice. We must therefore choose $d$ even smaller than .025 in order to employ $\tilde{E}_{\infty}$ on $D_{1}$, and we took $d=.0125$ in our experiments. Thus there are two disadvantages to using $\tilde{E}_{\infty}$ instead of $E_{\infty}$ : the elements contained in $D_{d} \backslash D$ will not be multiplied by extra factors of $h_{T}$, and we must sometimes choose $d$ smaller, thus potentially overemphasizing the pollution term.

Meshes resulting from using $E_{\infty}$ and $\tilde{E}_{\infty}$ to approximate $\left.\nabla u\right|_{D_{1}}$ are displayed in Figure 5 . When using $\tilde{E}_{\infty}$, we took $d=.0125$. Both pictures in Figure 5 show the same neighborhood of the origin, and both resulted in about the same actual error $\left(6.6 \times 10^{-5}\right.$ for $E_{\infty}$ and $6.3 \times 10^{-5}$ for $\left.\tilde{E}_{\infty}\right)$ and error estimate $\left(E_{\infty}=\tilde{E}_{\infty}=\right.$ $\left.1.57 \times 10^{-4}\right)$. However, the mesh resulting from employing $E_{\infty}$ had 23512 degrees of freedom versus 37393 for the mesh resulting from using $\tilde{E}_{\infty}$. The algorithm employing $\tilde{E}_{\infty}$ required about 1.35 times more degrees of freedom than that using 

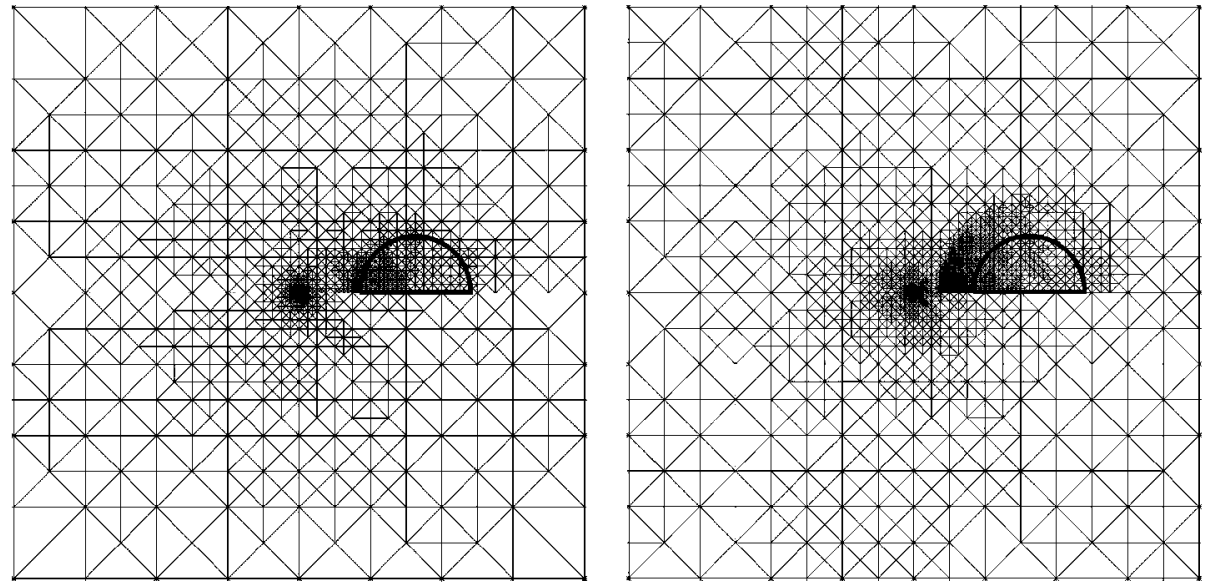

Figure 5. Meshes for calculation of $\left.\nabla u_{h}\right|_{D_{1}}$ using $E_{\infty}$ (left, 23512 DOF) and $\tilde{E}_{\infty}$ (right, $37393 \mathrm{DOF}$ ). Both are zoomed in toward the origin and display the square $\left[-\frac{1}{8}, \frac{1}{8}\right]^{2}$. The region $D_{1}$ is also marked.

$E_{\infty}$ to reach the tolerance .01, 1.45 times more to reach the tolerance .001, 1.54 times more to reach the tolerance .0001 , and 1.65 times more to reach the tolerance .00001. Thus the weighted estimator has a clear performance advantage over the unweighted estimator, as one would expect. It is also clear from Figure 5 that $\tilde{E}_{\infty}$ causes much heavier refinement in $D_{1, d} \backslash D$. Finally note that both meshes display clearly separated regions of high refinement near the origin and near $D_{1}$.

5.3. Experiment illustrating Corollary 3.2. Corollary 3.2 states that we may apply weighted estimators near convex corners of $\Omega$, but only with one power of
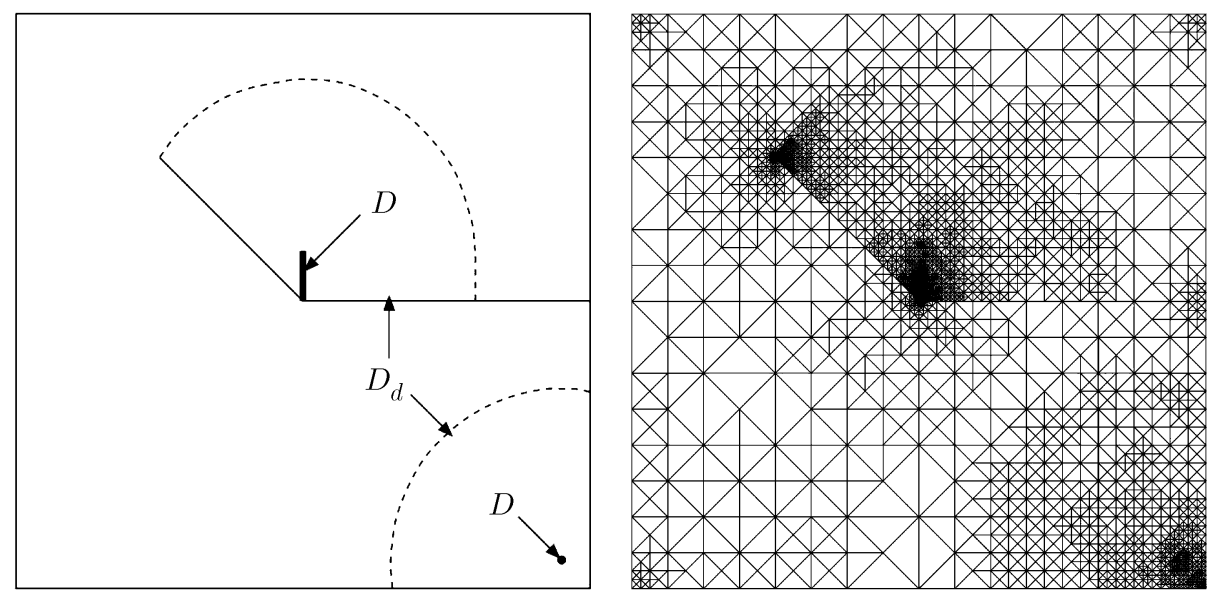

Figure 6. Left: the domain $\Omega_{2} ; D$ is represented by the thick line segment and dot. Right: mesh with 44983 DOF resulting from using cubic elements and $E_{\infty}$. 
the weight $\sigma_{D}$. In order to illustrate this fact, we conducted a simple experiment on the domain $\Omega_{2}$ pictured in Figure 6. Here $\Omega_{2}$ is a square with 2 connected line segments removed, that is, $\Omega_{2}$ is a crack domain where the crack itself consists of two connected line segments. We took $D=\{(.9,-.9)\} \cup\{0\} \times\left(0, \frac{1}{6}\right]$ (i.e., the union of a line segment and a point) in order to emphasize that $D$ need not be connected or a subdomain. In fact, it may be chosen as any subset of $\Omega_{2}$. Also, $d=.6$. The unknown solution $u \in H_{0}^{1}\left(\Omega_{2}\right)$ of $-\Delta u=1$ in $\Omega_{2}, u=0$ on $\partial \Omega_{2}$ was approximated on $D$ using cubic elements and the estimator $E_{\infty}$. The mesh resulting from approximating $\left.\nabla u\right|_{D}$ to a tolerance of $10^{-5}$ using the error estimator

$$
E_{\infty}=\max _{T \in \mathcal{T}} \max \left(C_{\text {app }}(r) \tilde{\sigma}_{D}(T), C_{\infty}(r) \frac{h_{T}}{d}\right) \mathcal{E}_{T}
$$

derived from Corollary 3.2 is shown in Figure 6. Four areas of heavy mesh refinement are clear: one near the crack tip $(-.5, .5)$, two at the origin (one on each side of the crack), and one near the point $(.9,-.9) \in D$. Thus the heaviest refinement occured at the re-entrant corners and near $D$, as was expected.

\section{ACKNOWLEDGMENT}

The author would like to thank an anonymous referee for several helpful comments which improved the presentation of this work.

\section{REFERENCES}

[BM87] I. BABUŠKa AND A. Miller, A feedback finite element method with a posteriori error estimation. I. The finite element method and some basic properties of the a posteriori error estimator, Comput. Methods Appl. Mech. Engrg., 61 (1987), pp. 1-40. MR.0880421(88d:73036)

[DDP00] E. DARI, R. G. DuRÁN, AND C. PADRA, Maximum norm error estimators for threedimensional elliptic problems, SIAM J. Numer. Anal., 37 (2000), pp. 683-700 (electronic). MR 1740762 (2001b:65120)

[Da88] M. DAuge, Elliptic boundary value problems on corner domains, vol. 1341 of Lecture Notes in Mathematics, Springer-Verlag, Berlin, 1988. MR.0961439 (91a:35078)

[Da92] Neumann and mixed problems on curvilinear polyhedra, Integral Equations Operator Theory, 15 (1992), pp. 227-261. MR.1147281 (93e:35025)

[De] A. Demlow, Localized pointwise a posteriori estimates for gradients of piecewise linear finite element approximations to second-order quasilinear elliptic problems, SIAM J. Numer. Anal. (to appear).

[DM95] G. Dolzmann and S. MülLer, Estimates for Green's matrices of elliptic systems by $L^{p}$ theory, Manuscripta Math., 88 (1995), pp. 261-273. MR1354111 (96g:35054)

[DR98] W. DöRfler AND M. RumpF, An adaptive strategy for elliptic problems including a posteriori controlled boundary approximation, Math. Comp., 67 (1998), pp. 13611382. MR.1489969 (99b:65141)

[GT98] D. Gilbarg And N. S. Trudinger, Elliptic Partial Differential Equations of Second Order, Springer-Verlag, Berlin, 2nd ed., 1998. MR 1814364 (2001k:35004)

[GW82] M. Grüter AND K.-O. Widman, The Green function for uniformly elliptic equations, Manuscripta Math., 37 (1982), pp. 303-342. MR0657523(83h:35033)

[HSWW01] W. Hoffmann, A. H. Schatz, L. B. Wahlbin, and G. Wittum, Asymptotically exact a posteriori estimators for the pointwise gradient error on each element in irregular meshes. I. A smooth problem and globally quasi-uniform meshes., Math. Comp., 70 (2001), pp. 897-909. MR1826572 (2002a:65178)

[Kr69] J. P. KrasovskiI, Properties of Green's functions and generalized solutions of elliptic boundary value problems, Soviet Math. Dokl., 10 (1969), pp. 54-58. MR0237956 $(38: 6233)$ 
[LN03] X. LiAO AND R. H. NochetTo, Local a posteriori error estimates and adaptive control of pollution effects, Numer. Methods Partial Differential Equations, 19 (2003), pp. 421-442. MR1980188 (2004c:65130)

[NS74] J. A. Nitsche AND A. H. Schatz, Interior estimates for Ritz-Galerkin methods, Math. Comp., 28 (1974), pp. 937-958. MR0373325 (51:9525)

[Noc95] R. H. Nochetto, Pointwise a posteriori error estimates for elliptic problems on highly graded meshes, Math. Comp., 64 (1995), pp. 1-22. MR.1270622 (95c:65172)

[NSSV] R. H. Nochetto, A. Schmidt, K. G. Siebert, and A. Veeser, Pointwise a posteriori error estimates for monotone semilinear problems, preprint.

[NSV03] R. H. Nochetto, K. G. Siebert, And A. Veeser, Pointwise a posteriori error control for elliptic obstacle problems, Numer. Math., 95 (2003), pp. 163-195. MR1993943 (2004g:49055)

[Sch00] A. H. SCHATZ, Pointwise error estimates and asymptotic error expansion inequalities for the finite element method on irregular grids. II. Interior estimates, SIAM J. Numer. Anal., 38 (2000), pp. 1269-1293 (electronic). MR.1786140 (2001i:65119)

[SW79] A. H. Schatz AND L. B. Wahlbin, Maximum norm estimates in the finite element method on plane polygonal domains. II. Refinements, Math. Comp., 33 (1979), pp. 465-492. MR0502067 (58:19233b)

[SW95] A. H. SChatz AND L. B. WAhLBIN, Interior maximum-norm estimates for finite element methods, Part II, Math. Comp., 64 (1995), pp. 907-928. MR1297478|(95j:65143)

[SS00] A. Schmidt ANd K. G. Siebert, ALBERT-software for scientific computations and applications, Acta Math. Univ. Comenian. (N.S.), 70 (2000), pp. 105-122. MR.1865363

[SS05] A. SCHMidt AND K. G. Siebert, Design of adaptive finite element software, vol. 42 of Lecture Notes in Computational Science and Engineering, Springer-Verlag, Berlin, 2005. The finite element toolbox ALBERTA, with 1 CD-ROM (Unix/Linux). MR 2127659(2005i:65003)

[SZ90] L. R. SCOTT AND S. ZHANG, Finite element interpolation of nonsmooth functions satisfying boundary conditions, Math. Comp., 54 (1990), pp. 483-493. MR1011446 (90j:65021)

[Ver94] R. VERFÜRTH, A posteriori error estimation and adaptive mesh-refinement techniques, in Proceedings of the Fifth International Congress on Computational and Applied Mathematics (Leuven, 1992), vol. 50, 1994, pp. 67-83. MR.1284252 (95c:65171)

Abteilung für Angewandte Mathematik, Hermann-Herder-Str. 10, 79104 Freiburg, Germany

E-mail address: demlow@mathematik.uni-freiburg.de 